\title{
Identifying gas composition changes to which non-injective gas sensor arrays are unresponsive
}

\author{
Nickolas Gantzler ${ }^{\dagger, *}$, Adrian Henle ${ }^{\ddagger * *}$, Praveen Thallapally ${ }^{\llbracket}$, \\ Xiaoli Fern ${ }^{\S}$, Cory M. Simon ${ }^{\ddagger}$ \\ $\dagger$ Department of Physics. Oregon State University. Corvallis, OR. \\ ${ }^{\ddagger}$ School of Chemical, Biological, and Environmental Engineering. Oregon State \\ University. Corvallis, OR. \\ IPacific Northwest National Laboratory. Richland, WA. \\ $\S$ School of Electrical Engineering and Computer Science. Oregon State University. \\ Corvallis, OR, USA \\ E-mail: cory.simon@oregonstate.edu \\ *equal contribution \\ 17 June 2021
}

\begin{abstract}
Metal-organic frameworks (MOFs) have great prospects as recognition elements for gas sensors owing to their adsorptive sensitivity and selectivity. A gravimetric, MOF-based sensor functions by measuring the mass of gas adsorbed in a thin film of MOF. Changes in the gas composition are expected to produce detectable changes in the mass of gas adsorbed in the MOF.

In practical settings, multiple components of the gas adsorb into the MOF and contribute to the sensor response. As a result, there are typically many distinct gas compositions that produce the same single-sensor response. The response vector of a gas sensor array places more constraints on the gas composition. Still, if the number of degrees of freedom in the gas composition is greater than the number of MOFs in the sensor array, the map from gas compositions to response vectors will be non-injective (many-to-one).

Here, we outline a mathematical method to determine undetectable changes in gas composition to which non-injective gas sensor arrays are unresponsive. This is important for understanding their limitations and vulnerabilities. We focus on gravimetric, MOF-based gas sensor arrays. Our method relies on a mixed-gas adsorption model in the MOFs comprising the sensor array, which gives the mass of gas adsorbed in each MOF as a function of the gas composition. The singular value decomposition of the Jacobian matrix of the adsorption model uncovers (i) the unresponsive directions and (ii) the responsive directions, ranked by sensitivity, in gas composition space. We illustrate the identification of unresponsive subspaces and ranked responsive directions for gas sensor arrays based on Co-MOF-74 and HKUST-1 aimed at quantitative sensing of $\mathrm{CH}_{4} / \mathrm{N}_{2} / \mathrm{CO}_{2} / \mathrm{C}_{2} \mathrm{H}_{6}$ mixtures (relevant to the natural gas industry).
\end{abstract}




\section{Introduction}

Gas sensors [1] have a wide range of applications in the chemical industry and in emerging domains such as air quality monitoring [2], diagnosis of disease [3], food quality assessment [4], detection of chemical warfare agents and explosives [5,6], and crop monitoring [7].

Metal-organic frameworks (MOFs) $[8]$ are nanoporous materials with high prospects as recognition elements for enhanced gas sensors

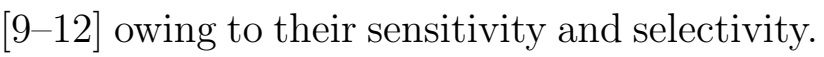
MOFs offer large internal surface areas and, often, expose functional groups and open metal sites to the pore space. As a result, gas molecules concentrate inside the pores of the MOF by (selectively) adsorbing onto the pore walls. The amount of gas adsorbed in a MOF at thermodynamic equilibrium depends upon the chemical potential of each component in the gas phase (and the temperature). Therefore, observation of the total mass of gas adsorbed in a MOF [13] - or some other property that depends on the gas adsorbed in the MOF, such as electrical resistance 14, 15], color [16], luminescence 17, 18], or strain 19,20 - provides information about the composition of the gas. Fig. 1 illustrates a miniaturized gravimetric, MOF-based sensor composed of a quartz crystal microbalance (QCM) coated with a thin film of $\mathrm{MOF}$ 21,22 - a surface mounted MOF (SURMOF) [23]. Nanogram-scale changes in the adsorbed mass of gas in the MOF film can be inferred from changes in the resonance frequency of the piezoelectric quartz crystal, forced by an alternating voltage [9,10]. Several gravimetric, MOF-based sensors have been experimentally demonstrated using QCMs [13, 24,28 and surface acoustic wave devices (SAWs) 2931 as signal transducers.

Cross-sensitivity plagues gas sensors in

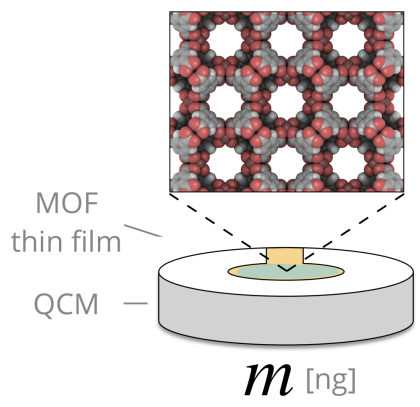

Figure 1: A gravimetric sensor comprised of a thin film of metal-organic framework (MOF) 23 (the recognition element), in this case HKUST-1 [32], mounted on a quartz crystal microbalance (QCM) 22,33] (the signal transducer). The QCM measures the (total) adsorbed mass of gas, $m$, in the thin film of MOF. The QCM-MOF functions as a sensor because changes in the gas composition, $\mathbf{x}$, are expected to cause changes in $m$.

practical applications where the gas contains multiple components that vary in concentration [34]. In the case of MOF-based gas sensors, interfering gas species, in addition to the analyte species, adsorb onto the MOF and contribute to the response (i.e. the total adsorbed mass of gas). As a consequence, the inverse problem [35] of inferring the concentration of the analyte from a single-sensor response is underdetermined [36]. To address the problem of cross-sensitivity, one might propose to design a MOF with a very high adsorptive selectivity for the analyte. However, this strategy may be difficult or impossible, and it requires a different MOF highly tailored to each sensing task.

Gas sensor arrays tackle the issue of crosssensitivity by monitoring the response of a diverse set of (likely, cross-sensitive) MOFs [37, 38], analogous to mammalian olfactory receptors 39]. Each response of a crosssensitive MOF, though having contributions from the analyte and interferents, places one 


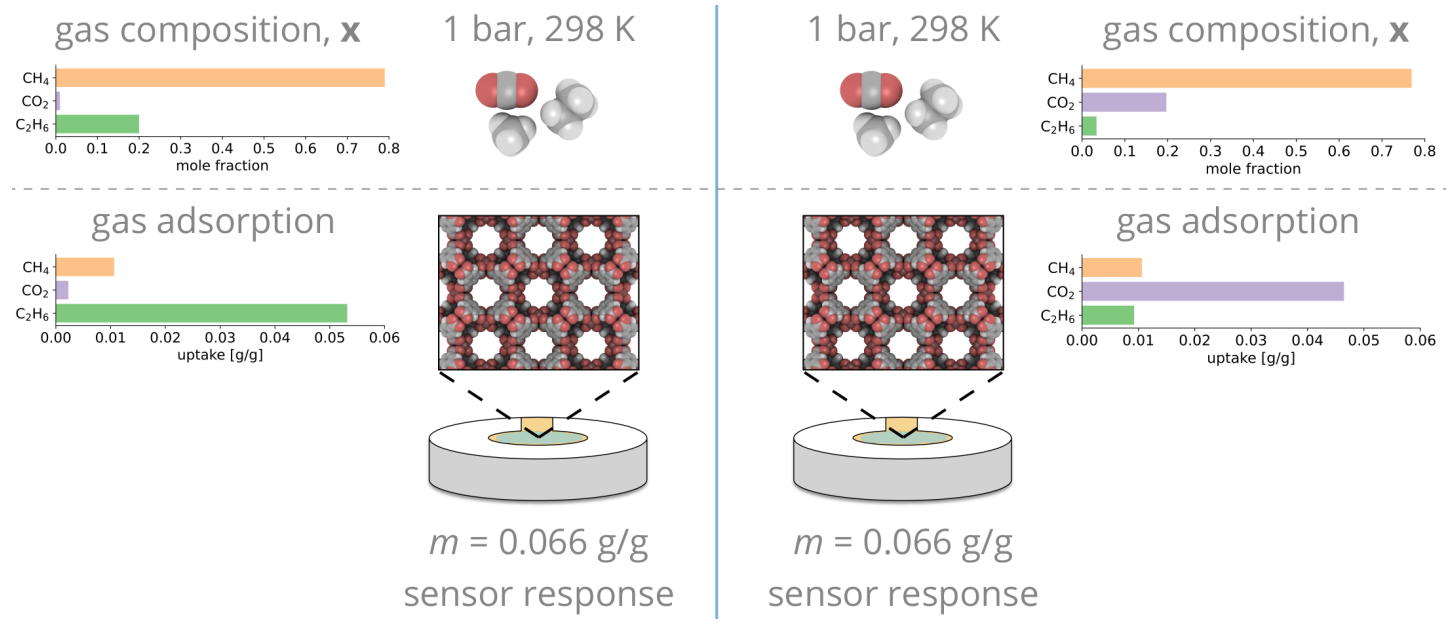

Figure 2: Illustrating a gas composition change to which a non-injective, gravimetric, MOFbased gas sensor is unresponsive. A single QCM-HKUST-1 sensor is immersed in a gas mixture of two different compositions: on the left, a predominantly $\mathrm{CH}_{4} / \mathrm{C}_{2} \mathrm{H}_{6}$ mixture; on the right, a predominantly $\mathrm{CH}_{4} / \mathrm{CO}_{2}$ mixture. The response of the sensor to the two distinct gas compositions is identical; hence, the sensor cannot be used to distinguish between these two compositions. The total mass of gas adsorbed in the HKUST-1 film remains the same upon changing from the $\mathrm{CH}_{4} / \mathrm{C}_{2} \mathrm{H}_{6}$ to $\mathrm{CH}_{4} / \mathrm{CO}_{2}$ mixture because the desorption of $\mathrm{C}_{2} \mathrm{H}_{6}$ balances [in mass] the adsorption of $\mathrm{CO}_{2}$, while the adsorption of $\mathrm{CH}_{4}$ remains approximately constant.

constraint on the gas composition. As a whole, the response vector of the gas sensor array may provide sufficient information to uniquely determine the gas composition. Typically, a machine learning algorithm is used to parse the high-dimensional response of a sensor array [40]. A few MOF-based gas sensor arrays have been experimentally demonstrated [14, 27, 41,43]. Still, even gas sensor arrays are susceptible to cross-sensitivity; the inverse problem (determine the gas composition from the sensor array response vector) could still be underdetermined - particularly, if the number of MOFs comprising the array is less than the number of degrees of freedom in the gas composition. This circumstance can arise (i) in an environment with highly complex gas mixtures and/or (ii) a sensor array is calibrated for gas mixtures with a specific set of components, but an unexpected, interfering component is introduced.

In this article, we outline a mathematical method to identify changes in the gas composition to which reversible $\$$ quantitative gas sensors /sensor arrays are unresponsive. Gas sensing can be viewed as the inverse problem of finding the gas composition consistent with the sensor response governed by a mixedgas adsorption model [35]. Owing to crosssensitivity, the mapping, under the adsorption model, from gas composition space to sensor array response space could be non-injective (many-to-one). Consequently, some gas composition changes are undetectable. By our defi-

$\ddagger$ Gas sensors based on physical adsorption in stable MOFs lacking adsorption/desorption hysteresis are reversible. Suppose the gas composition changes from (i) a reference composition to (ii) some new composition, then (iii) back to the reference composition. The equilibrium mass of gas adsorbed in the MOF will be the same in (i) and (iii). 
nition, when the gas composition changes in an unresponsive direction in composition space, the sensor response remains constant. When the gas composition changes in an unresponsive direction, the adsorption of one set of species balances (in mass) the desorption of another set of species, thus, keeping the sensor response fixed. These undetectable gas composition changes generally depend upon the initial composition. The identification of unresponsive directions of gas sensors/sensor arrays is important for understanding their limitations and vulnerabilities to adversarial attacks [44].

Specifically, we show that the singular value decomposition of the Jacobian matrix of the mapping from gas composition vectors to sensor response vectors is key to determining unresponsive directions and responsive directions, ranked by sensitivity, in composition space. In particular, unresponsive directions follow from the null space of the Jacobian. We illustrate this under the context of quantitative sensing for diluents and higher-hydrocarbons in natural gas [45 $\left(\mathrm{N}_{2}, \mathrm{CO}_{2}\right.$, and $\mathrm{C}_{2} \mathrm{H}_{6}$ in $\mathrm{CH}_{4}$ ) using gravimetric, MOF-based sensors comprised of HKUST-1 and Co-MOF-74. We use experimental gas adsorption data and ideal adsorbed solution theory [46, 47] to model the mass of adsorbed gas in each MOF in response to different gas compositions. Under several case studies, we visualize the unresponsive and ranked responsive directions together with the underlying mixed gas adsorption model.

Several computational and mathematical modeling approaches have been developed to design/analyze gas sensor arrays of MOFs 35, 36, 48 52. . Our work focuses on identifying the limitations of non-injective, MOF-based gas sensor arrays by elucidating directions in composition space in which the gas sensor is unresponsive to changes.

\section{A mathematical model of gas sensor arrays}

\subsection{Problem setup}

Suppose a gravimetric sensor array of $M$ MOFs aims to determine the composition of a gas (temperature, pressure fixed) with $G+1$ components.

The gas composition vector. Let $\mathbf{x} \in \mathbb{R}^{G}$ be the gas composition vector, with $x_{i}$ the mole fraction of component $i$ in the gas phase. We omit component $G+1$ of the gas from $\mathbf{x}$ since its mole fraction follows from $x_{G+1}=$ $1-\left(x_{1}+x_{2}+\cdots+x_{G}\right)$. Thus, the only constraints on $\mathbf{x}$ are $\sum_{i=1}^{G} x_{i} \leq 1$ and $\mathbf{x} \geq \mathbf{0}$. We assume the gas is held at constant pressure and temperature, implying (i) $\mathbf{x}$ fully specifies the thermodynamic state of the gas phase and (ii) $G$ is the number of degrees of freedom in the gas phase.

The sensor response vector. Let $\mathbf{m} \in \mathbb{R}^{M}$ be the sensor array response vector, with $m_{i}$ [g gas/g MOF] denoting the total mass of gas adsorbed in MOF $i$ at thermodynamic equilibrium, normalized by the mass of MOF in the sensor. We assume, as in practice, that each MOF is cross-sensitive. As a result, $m_{i}$ is comprised of contributions from each of the $G+1$ components of the gas phase.

The adsorption function. Mathematically, we view gas sensor arrays as mapping from gas composition space $\mathcal{X}:=\mathbb{R}^{G}$ to sensor response space $\mathcal{M}:=\mathbb{R}^{M}$ 36, 53]. The adsorption function $a: \mathbf{x} \mapsto \mathbf{m}$ maps each gas composition vector $\mathrm{x} \in \mathcal{X}$ to a sensor response vector $\mathbf{m} \in \mathcal{M}$. I.e., $\mathbf{m}=a(\mathbf{x})$ is a model for the total mass of gas adsorbed in each MOF (at thermodynamic equilibrium) as a 
function of the gas composition. We assume $a(\mathbf{x})$ is a continuously differentiable function, disallowing adsorption/desorption hysteresis, gas-induced first-order structural transitions, chemical transformations, and condensation of adsorbates in the pores.

A model of the adsorption function $a(\mathbf{x})$ could be constructed from adsorption data $\mathcal{D}=\left\{\left(\mathbf{x}_{i}, \mathbf{m}_{i}\right)\right\}_{i=1}^{n}$, with $n$ the number of gas exposure experiments, through various methods including:

- interpolation of $\mathcal{D}$.

- a statistical mechanical model of gas adsorption [54 whose parameters are identified using $\mathcal{D}$.

- a statistical machine learning model trained on $\mathcal{D}$.

- ideal adsorption solution theory (IAST) [55] applied to pure-component adsorption models constructed from $\mathcal{D}$.

The source of $\mathcal{D}$ could be experimental gas adsorption measurements and/or grandcanonical Monte Carlo molecular simulations of gas adsorption [56, 57] in the MOFs.

While we write the adsorption function $a(\mathbf{x})$ as a vector-valued function, the mass of gas adsorbed in each MOF is independent. In other words, we can independently build the adsorption model for each MOF $i$ in the array, $m_{i}=a_{i}(\mathbf{x})$.

Gas sensing as an inverse problem [35]. The forward problem is to predict the response of the sensor array $\mathbf{m}$ given the gas composition $\mathbf{x}$. The solution is to evaluate the adsorption model $\mathbf{m}=a(\mathbf{x})$. The inverse problem arises in gas sensing: we wish to predict the gas composition $\mathbf{x}$ given the sensor response $\mathbf{m}$ using the adsorption model $\mathbf{m}=a(\mathbf{x})$. While the forward problem always has a unique solution, the inverse problem may have a unique solution, no solution, or many solutions.

\subsection{Non-injective gas sensor arrays with underdetermined inverse problems}

In this work, we consider gas sensor arrays comprised of fewer MOFs than degrees of freedom in the gas composition $(M<G)$. The mapping $a: \mathbf{x} \mapsto \mathbf{m}$ under a sensor array with $M<G$ is generally non-injective (manyto-one). Consequently, the inverse problem of determining the gas composition $\mathbf{x}$ from the sensor response $\mathbf{m}$ is underdetermined; typically, many gas compositions can produce the observed response. In the inverse problem, observation of each MOF's response, generally, provides one independent constraint, $m_{i}=$ $a_{i}(\mathbf{x})$, on the gas composition. For $M<G$, the response of the sensor array places an insufficient number of constraints on the gas composition to uniquely determine it.

As a result of their (view 1) noninjective mapping $a: \mathbf{x} \mapsto \mathbf{m}$ and (view 2) underdetermined inverse problems, gas sensor arrays with fewer MOFs than degrees of freedom in the gas $(M<G)$ suffer from unresponsiveness to some changes in gas composition: despite the change in composition, the sensor response remains constant, and hence the composition change is not detected.

\section{Unresponsive and ranked responsive directions of non-injective gas sensors}

Non-injective gas sensors contain fewer MOFs than degrees of freedom in the gas composition. They are vulnerable to blindness: the sensor is unresponsive to certain gas composition changes. Our work focuses on identifying 
these vulnerabilities in non-injective gas sensors. Moreover, the sensor is more sensitive to certain gas composition changes than others (of the same magnitude). We show that the singular value decomposition of the Jacobian matrix of the adsorption function $a(\mathbf{x})$ is key to identifying directions in composition space to which the sensor is most-, least-, and unresponsive.

\subsection{The operating point $\mathbf{x}_{o p}$ as a reference}

We will define unresponsive and ranked responsive directions in reference to some steady-state, reference gas composition, or operating point $\mathbf{x}_{o p} \in \mathbb{R}^{G} \cdot \mathbf{m}_{o p}:=a\left(\mathbf{x}_{o p}\right)$ is the sensor response at this operating point.

\subsection{The unresponsive locus $\mathcal{B}$}

The unresponsive locus $\mathcal{B} \subset \mathcal{X}$ associated with an operating point $\mathbf{x}_{o p}$ is the set of gas compositions such that the sensor response remains constant at $\mathbf{m}_{o p}$ :

$$
\mathcal{B}:=\left\{\mathbf{x}: a(\mathbf{x})=\mathbf{m}_{o p}, \mathbf{x} \in \mathcal{X}\right\}
$$

The unresponsive locus is an intersection of the level sets of the adsorption functions for each MOF in the sensor array, $\mathcal{B}=\cap_{i=1}^{M}\{\mathbf{x}$ : $\left.a_{i}(\mathbf{x})=m_{i, o p}\right\}$. By definition, the sensor array cannot distinguish between gas compositions in the unresponsive locus. A change in the gas composition from $\mathbf{x}_{o p} \in \mathcal{B}$ to $\mathbf{x}^{\prime} \in \mathcal{B}$ is undetectable despite $\mathbf{x}^{\prime} \neq \mathbf{x}_{o p}$. Fundamentally, the response of a sensor array composed of cross-sensitive MOFs remains constant under the composition change from $\mathbf{x}_{o p}$ to $\mathbf{x}^{\prime}$ because the adsorption of one set of species balances [in mass] the desorption of another set of species (see Fig. 2). A trivial example of a non-trivial unresponsive locus is when all MOFs do not adsorb a particular component of the gas.

\subsection{Linear approximation of $a(\mathbf{x})$ near the} operating point $\mathbf{x}_{o p}$

Let us make a linear approximation $\tilde{a}(\mathbf{x})$ of the adsorption function $a(\mathbf{x})$ valid near the operating point $\mathbf{x}_{o p}$. A first-order Taylor expansion of $a(\mathbf{x})$ about $\mathbf{x}_{o p}$ gives:

$$
a(\mathbf{x}) \approx a\left(\mathbf{x}_{o p}\right)+\mathbf{J}\left(\mathbf{x}_{o p}\right)\left(\mathbf{x}-\mathbf{x}_{o p}\right)=: \tilde{a}(\mathbf{x}),
$$

with $\mathbf{J}=\mathbf{J}(\mathbf{x})$ the $M \times G$ Jacobian matrix of $a(\mathbf{x})$ with $J_{i j}:=\frac{\partial m_{i}}{\partial x_{j}}$. For a non-injective gas sensor array $(M<G)$, $\mathbf{J}$ is fat. From a geometric viewpoint, the approximator $m_{i} \approx$ $\tilde{a}_{i}(\mathbf{x})$ is a hyperplane tangent to the surface $a_{i}(\mathbf{x})$ and passing through $m_{i, o p}$.

\subsection{The (local) unresponsive subspace of composition space}

We define the (locally valid) unresponsive subspace $\tilde{\mathcal{B}} \subset \mathcal{X}$ of a sensor array associated with operating point $\mathbf{x}_{o p}$ as the directions in composition space such that, for small changes in the gas composition in these directions, the sensor response remains approximately constant at $\mathbf{m}_{o p}$. Thus, for a unit vector $\Delta \mathbf{x} \in \tilde{\mathcal{B}}, a\left(\mathbf{x}_{o p}+\epsilon \Delta \mathbf{x}\right) \approx a\left(\mathbf{x}_{o p}\right)$ with $\epsilon$ a small number. Invoking the linear approximation $\tilde{a}(\mathbf{x})$ in eqn. 2, the local unresponsive subspace is given by the null space of the Jacobian matrix $\mathbf{J}\left(\mathbf{x}_{o p}\right)$ :

$$
\begin{aligned}
\tilde{\mathcal{B}} & :=\left\{\Delta \mathbf{x}: \mathbf{J}\left(\mathbf{x}_{o p}\right) \Delta \mathbf{x}=\mathbf{0}, \Delta \mathbf{x} \in \mathcal{X}\right\} \\
& \Longrightarrow \tilde{a}\left(\mathbf{x}_{o p}+\Delta \mathbf{x}\right)=\tilde{a}\left(\mathbf{x}_{o p}\right)=\mathbf{m}_{o p} \quad \forall \Delta \mathbf{x} \in \tilde{\mathcal{B}} .
\end{aligned}
$$

The null space of $\mathbf{J}$ will be non-trivial provided $G>M$. I.e, sensor arrays such that $M<G$ possess non-trivial unresponsive subspaces due to the non-injective nature of the mapping $\tilde{a}: \mathbf{x} \mapsto \mathbf{m}$. 


\subsection{The sensitive direction in composition} space

The sensor response is most sensitive to gas composition changes in the direction of:

$$
\Delta \mathbf{x}_{s}:=\underset{\Delta \mathbf{x}:\|\Delta \mathbf{x}\|=\epsilon}{\arg \max }\left\|\tilde{a}\left(\mathbf{x}_{o p}+\Delta \mathbf{x}\right)-\tilde{a}\left(\mathbf{x}_{o p}\right)\right\| .
$$

Of all gas composition changes $\Delta \mathbf{x}$ from $\mathbf{x}_{o p}$ that are small $(\epsilon>0)$ in magnitude, those in the sensitive direction of $\Delta \mathbf{x}_{s}$ elicit the largestmagnitude change in the response of the sensor array.

\subsubsection{The singular value decomposition} (SVD) of J. The singular value decomposition (SVD) [58,59 of the $M \times G$ Jacobian matrix $\mathbf{J}$ factorizes it as:

$$
\mathbf{J}=\mathbf{U} \boldsymbol{\Sigma} \mathbf{V}^{\top}
$$

where $\mathbf{U}$ is a $M \times M$ orthogonal matrix, $\mathbf{\Sigma}$ is an $M \times G$ diagonal matrix with singular values $\sigma_{1} \geq \sigma_{2} \geq \cdots \geq \sigma_{M}$ listed down the diagonal, and $\mathbf{V}$ is a $G \times G$ orthogonal matrix. The left singular vector $\mathbf{u}_{i} \in \mathcal{M}$ is column $i$ of $\mathbf{U}$ and lies in sensor response space. The right singular vector $\mathbf{v}_{i} \in \mathcal{X}$ is column $i$ of $\mathbf{V}$ and lies in gas composition space. The $M$ left ( $G$ right) singular vectors form an orthonormal basis for sensor response (gas composition) space. Provided the MOFs are distinct, it is extremely likely that $\mathbf{J}$ is full-rank $(\operatorname{rank} M) \S$. so we assume $\sigma_{M}>0$ in this work.

Seen by $\mathbf{J V}=\mathbf{U} \boldsymbol{\Sigma}$ :

$$
\mathbf{J}_{i}= \begin{cases}\sigma_{i} \mathbf{u}_{i} & i \in\{1,2, \ldots, M\} \\ \mathbf{0} & i \in\{M+1, M+2, \ldots, G\}\end{cases}
$$

Therefore, under the linear approximation $\tilde{a}(\mathbf{x})$ in eqn. 2, the sensor array is:

$\S \mathbf{J}$ could, however, be ill-conditioned 35 .
- responsive to gas composition changes in the direction belonging to the subspace spanned by the first $M$ right singular vectors $\mathbf{v}_{1}, \mathbf{v}_{2}, \ldots, \mathbf{v}_{M}$. They are ranked by the magnitude of the sensor array response for composition changes of fixed magnitude.

- unresponsive to gas composition changes in the direction belonging to the subspace spanned by the last $G-M$ right singular vectors $\left\{\mathbf{v}_{M+1}, \mathbf{v}_{M+2}, \ldots, \mathbf{v}_{G}\right\}$. This subspace is the null space of $\mathbf{J}$.

Hence,

- the sensitive direction of the gas sensor array, $\Delta \mathbf{x}_{s}$ in eqn. 5, is described by the first right singular vector $\mathbf{v}_{1}$ of the Jacobian matrix J. The gas sensor array is responsive, but less sensitive, to composition changes in the remaining directions $\mathbf{v}_{2}, \mathbf{v}_{3}, \ldots, \mathbf{v}_{M}$.

- the local unresponsive subspace $\tilde{\mathcal{B}}$ is spanned by the last (unranked) $G-M$ right singular vectors $\left\{\mathbf{v}_{M+1}, \mathbf{v}_{M+2}, \ldots, \mathbf{v}_{G}\right\}$ of $\mathbf{J}$.

We emphasize that the sensitive and unresponsive directions are (i) depend on the operation gas composition $\mathbf{x}_{o p}$ and (ii) pertain to small changes in the gas composition since they rely on the linear approximation of the generally non-linear function $a(\mathbf{x})$ in eqn. 2 .

We remark on the uniqueness of the SVD in eqn. 6. The singular values are unique. The left and right singular vectors associated with the distinct, non-zero singular values are unique, up to both corresponding vectors being multiplied by -1 . The right singular vectors spanning the null space are much more arbitrary in the sense that they can be any orthonormal basis for the null space.

Note, if the response vector is recorded in units $\mathrm{g}$ gas instead of $\mathrm{g}$ gas/g MOF, (i) 
the responsive and unresponsive subspaces do not change, but (ii) the ranked responsive directions that span the responsive subspace do change if different masses of MOF are used in each sensor.

\subsection{Special case: linear adsorption model}

Suppose gas adsorption in each MOF of the array is described by Henry's law:

$$
m_{i}=\sum_{j=1}^{G+1} H_{i, j} P x_{j},
$$

with $P$ the total pressure [bar] of the gas phase and $H_{i, j}$ the Henry coefficient [g gas/g MOF-bar)] of gas $j$ in MOF $i$. Imposing the constraint that the mole fractions $\left\{x_{j}\right\}$ sum to one, the adsorption model for the sensor array is linear:

$$
\mathbf{m}=a(\mathbf{x})=\mathbf{A} \mathbf{x}+\mathbf{b},
$$

with $A_{i j}:=P\left(H_{i, j}-H_{i, G+1}\right), b_{i}:=H_{i, G+1}$, and $\mathbf{x}:=\left[x_{1}, x_{2}, \ldots, x_{G}\right]$. Under this special case, the Jacobian matrix $\mathbf{J}=\mathbf{A}$ does not depend on a reference gas composition $\mathbf{x}_{o p}$. Therefore, the unresponsive and responsive subspaces are independent of $\mathbf{x}_{o p}$, too.

\section{Summarizing with a toy example}

We illustrate the identification of the unresponsive and ranked responsive directions with a toy example: a sensor array of $M=2$ distinct MOFs aimed at sensing a quaternary mixture at fixed temperature and pressure $(G=3)$. See Fig. 3a.

Fig. 3c illustrates the singular value decomposition of a $M=2 \times 3=G$ Jacobian matrix $\mathbf{J}=\mathbf{J}\left(\mathbf{x}_{o p}\right)$ at some operating point $\mathbf{x}_{o p}$. The three orthonormal right singular vectors $\left\{\mathbf{v}_{1}, \mathbf{v}_{2}, \mathbf{v}_{3}\right\}$ lie in $G=3$-dimensional composition space, while the two orthonormal left singular vectors $\left\{\mathbf{u}_{1}, \mathbf{u}_{2}\right\}$ lie in $M=2$ dimensional response space. The Jacobian matrix maps the right-singular vectors into response space as follows:

$$
\begin{aligned}
& \mathbf{J}_{\mathbf{v}_{1}}=\sigma_{1} \mathbf{u}_{1} \\
& \mathbf{J}_{\mathbf{v}_{2}}=\sigma_{2} \mathbf{u}_{2} \\
& \mathbf{J}_{\mathbf{v}_{3}}=\mathbf{0} .
\end{aligned}
$$

Provided the MOFs are distinct, the two rows of $\mathbf{J}$ are highly unlikely to point in exactly the same direction; thus, we safely assume $\mathbf{J}$ is fullrank, i.e., $\sigma_{2}>0$. Eqns. 10 and 11 indicate the sensor is responsive to gas composition changes in the direction of $\mathbf{v}_{1}$ and $\mathbf{v}_{2}$ but more sensitive to changes in the direction of $\mathbf{v}_{1}\left(\sigma_{1} \geq \sigma_{2}\right.$ by definition, but likely $\left.\sigma_{1}>\sigma_{2}\right)$. Eqn. 12 indicates the null space of the Jacobian matrix is non-trivial and spanned by $\mathbf{v}_{3}$. Therefore, the linear map $\tilde{a}: \mathbf{x} \mapsto \mathbf{m}$ is non-injective, as a consequence of $M<G$.

Consider small gas composition changes (of magnitude $\epsilon>0$ ) from $\mathbf{x}_{o p}$. The linear approximant $\tilde{a}(\mathbf{x})$ in eqn. 2 maps a sphere in $3 \mathrm{D}$ composition space centered at $\mathbf{x}_{o p}$ to a solid ellipse in 2D sensor response space [58 centered at $\mathbf{m}_{o p}$. See Fig. 3b. This follows from eqn. 2 and eqns. $10, \sqrt{12}$. The composition change in the sensitive direction, $\epsilon \mathbf{v}_{1}$ (green in Fig. 3b), elicits the largest sensor response-from $\mathbf{m}_{o p}$ to $\mathbf{m}_{o p}+\epsilon \sigma_{1} \mathbf{u}_{1}$ on the endpoint of the major axis of the ellipse. The sensor is responsive, but less sensitive, to composition changes in the direction of $\mathbf{v}_{2}$ (yellow in Fig. 3b); the composition change $\epsilon \mathbf{v}_{2}$ moves the sensor response by the vector $\epsilon \sigma_{2} \mathbf{u}_{2}$, placing it on the endpoint of the minor axis of the ellipse. The relative sensitivity of the response to composition changes in the direction of $\mathbf{v}_{1}$ vs. $\mathbf{v}_{2}$ depends on the singular values, $\sigma_{1}$ vs. $\sigma_{2}$. On the other hand, $\mathbf{v}_{3}$ is 
the unresponsive direction; the composition change $\epsilon \mathbf{v}_{3}$ (red in Fig. 3b) does not elicit a sensor response, and $\mathbf{m}$ remains at $\mathbf{m}_{o p}$ despite the change in gas composition. Using this toy sensor array, we therefore cannot detect small composition changes in the direction of $\mathbf{v}_{3}$ !

\section{Demonstration: natural gas sensing with gravimetric sensors based on HKUST-1 and Co-MOF-74}

We now demonstrate the identification of the unresponsive subspace and ranked responsive directions in gas composition space for a gravimetric, single-MOF sensor and twoMOF sensor array for diluents and higherhydrocarbons in natural gas 45]. Specifically, suppose the sensor (array) is immersed in a variable-composition gas mixture involving $\mathrm{N}_{2}$, $\mathrm{C}_{2} \mathrm{H}_{6}, \mathrm{CO}_{2}$, and $\mathrm{CH}_{4}$ at 1 bar and $298 \mathrm{~K}$. We consider two candidate MOFs as the recongition element(s), HKUST-1 32 and CoMOF-74 60] (see Fig. 4a).

In each case study below, the number of degrees of freedom in the gas composition $(G$, with $G+1$ the number of components in the gas) is greater than the number of MOFs $(M)$ comprising the sensor (array). Thus, (i) the sensor's mapping under $a$ from gas composition space to sensor response space is non-injective and (ii) the inverse problem to determine the gas composition from the sensor response is underdetermined. Accordingly, the sensor (array) will contain a non-trivial unresponsive subspace.

The data and Python code to reproduce all plots in our article are available at github. com/SimonEnsemble/unresponsive_direction in_sensors.

\subsection{The adsorption model $a(\mathbf{x})$, its Jacobian} matrix $\mathbf{J}$, and its SVD.

We construct an adsorption model for each MOF on the basis of experimentally measured, pure-component $\mathrm{CH}_{4}, \mathrm{CO}_{2}, \mathrm{C}_{2} \mathrm{H}_{6}$, and $\mathrm{N}_{2}$ adsorption data at or near $298 \mathrm{~K}$. Fig. 4b displays the adsorption isotherms and Tab. 1 provides the references.

Table 1: References for experimentally measured, equilibrium gas adsorption isotherms at $298 \mathrm{~K}$ (except $\mathrm{C}_{2} \mathrm{H}_{6}$, at $296 \mathrm{~K}$ ).

\begin{tabular}{ccccc} 
MOF & $\mathrm{CH}_{4}$ & $\mathrm{CO}_{2}$ & $\mathrm{C}_{2} \mathrm{H}_{6}$ & $\mathrm{~N}_{2}$ \\
\hline HKUST-1 & 61 & 62 & 63 & 64 \\
Co-MOF-74 & 65 & 65 & 63 & 65
\end{tabular}

We invoke ideal adsorbed solution theory (IAST) 47, 55 and use pyIAST 66 to implement the adsorption function $a(\mathbf{x})$. Sec. 2.1 explains that $a(\mathbf{x})$ gives the total mass of gas adsorbed in each MOF $[\mathrm{g} / \mathrm{g}]$ of the array as a function of the gas composition described by $\mathbf{x}$. IAST is a thermodynamic framework to predict mixed-gas adsorption from pure-component adsorption isotherms. Fig. $4 \mathrm{~b}$ shows the pure-component adsorption isotherm models fit to the experimental adsorption data and used as input for IAST calculations; the single-site Langmuir, dualsite (DS) Langmuir, and quadratic adsorption isotherm models fit the data well. N.b., though we write $\mathbf{m}=a(\mathbf{x})$, the adsorption model for each MOF in the array is independent; IAST provides $m_{i}=a_{i}(\mathbf{x})$ for each MOF in the array independently.

We compute the Jacobian matrix $\mathbf{J}=$ $\mathbf{J}\left(\mathbf{x}_{o p}\right)$ of $a(\mathbf{x})$ numerically via a second-order, centered finite difference using numdifftools; we compute the SVD of $\mathbf{J}$ using numpy. 


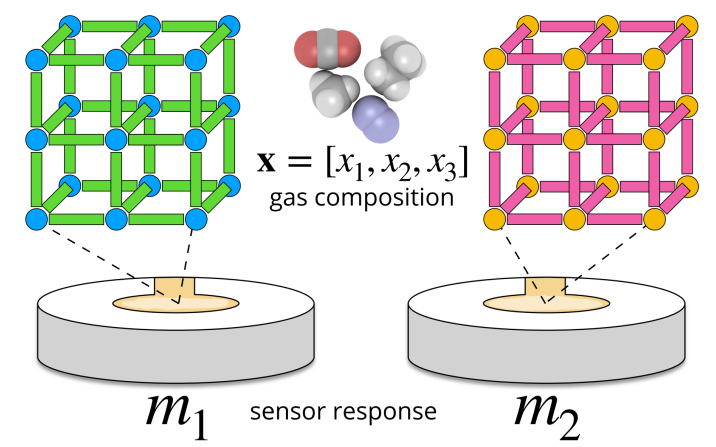

(a)
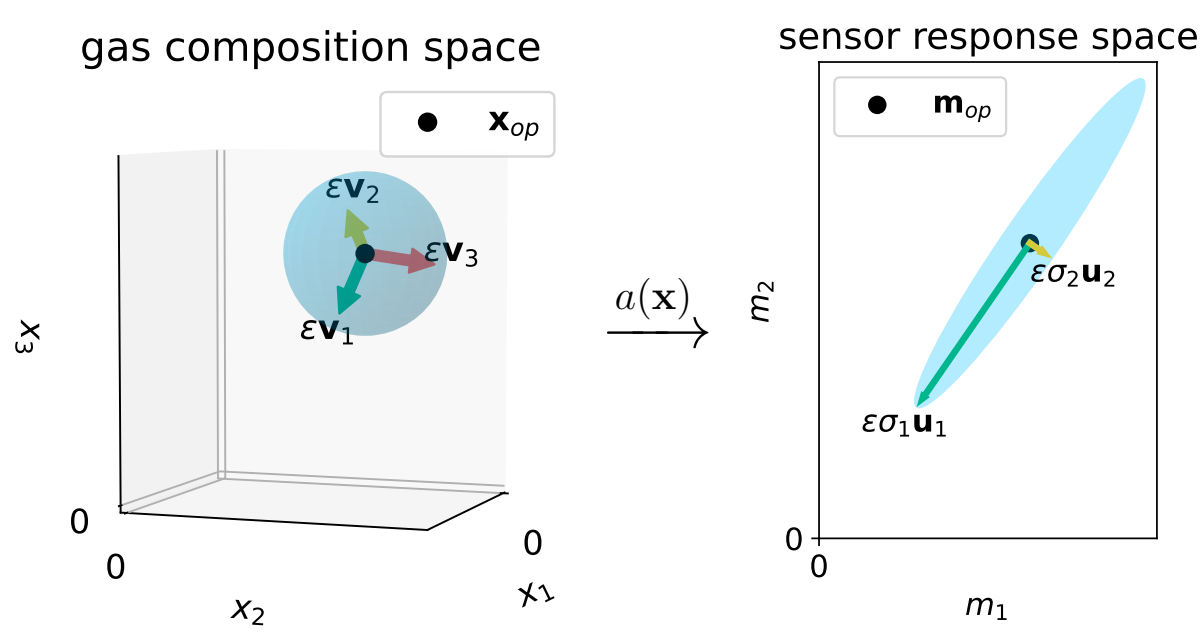

(b)

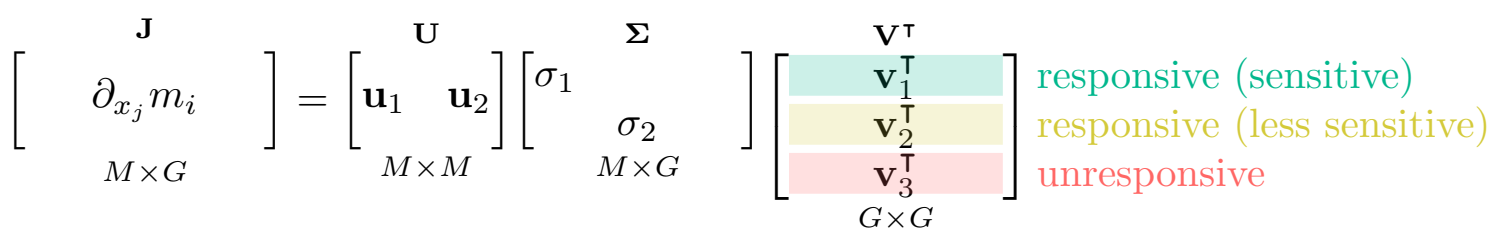

(c)

Figure 3: Elucidating the unresponsive and ranked responsive directions of a (toy) sensor array through the singular value decomposition (SVD) of the Jacobian matrix $\mathbf{J}$ of its adsorption function $a(\mathbf{x})$. (a) A gravimetric, two-MOF sensor array immersed in a four-component gas mixture at constant temperature and pressure but varying composition. The gas composition $\mathbf{x} \in \mathbb{R}^{3}$, while the sensor response $\mathbf{m} \in \mathbb{R}^{2}$. (b) The adsorption function $a(\mathbf{x})$ is a map $a: \mathbf{x} \mapsto \mathbf{m}$ from 3D gas composition space (left) to $2 \mathrm{D}$ sensor response space (right). The operating gas composition $\mathbf{x}_{o p}$ and associated response $\mathbf{m}_{o p}$ are shown as black points. Under the linear approximation of $a(\mathbf{x})$ valid locally around $\mathbf{x}_{o p}$ : The image of the sphere of small radius $\epsilon$ (blue, left) maps to the solid ellipse (blue, right) with semi-major and semi-minor axes given by $\epsilon \sigma_{1} \mathbf{u}_{1}$ and $\epsilon \sigma_{2} \mathbf{u}_{2}$, respectively. Gas composition changes $\epsilon \mathbf{V}_{1}, \epsilon \mathbf{v}_{2}, \epsilon \mathbf{v}_{3}$ form an orthogonal basis of composition space and are mapped to response changes $\epsilon \sigma_{1} \mathbf{u}_{1}, \epsilon \sigma_{2} \mathbf{u}_{2}, \mathbf{0}$, respectively. $\mathbf{v}_{3}$ describes the unresponsive direction, since small changes in composition in this direction do not alter the response. Small composition changes in the direction of $\mathbf{v}_{1}$ elicit the largest-magnitude response. (c) The singular value decomposition $\mathbf{J}=\mathbf{U} \boldsymbol{\Sigma} \mathbf{V}^{\top}$ gives the vectors described in (b). 

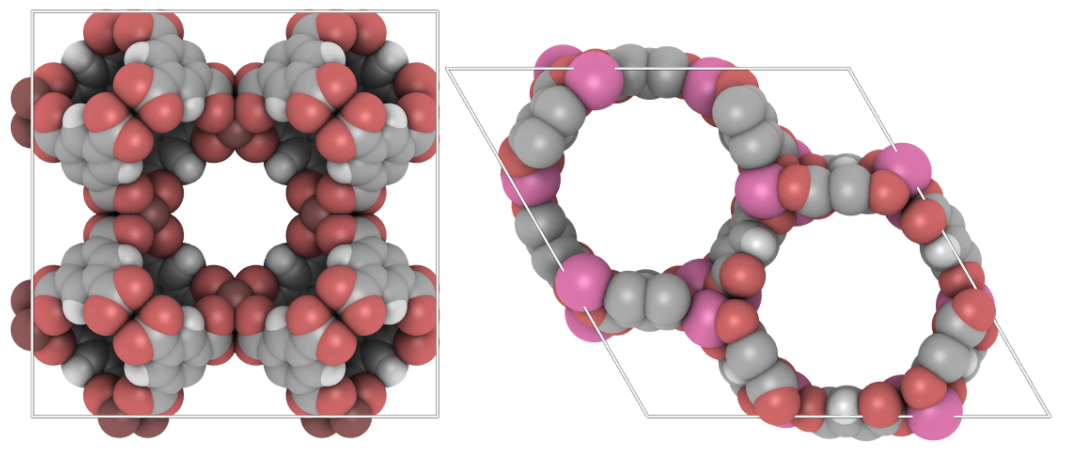

(a)
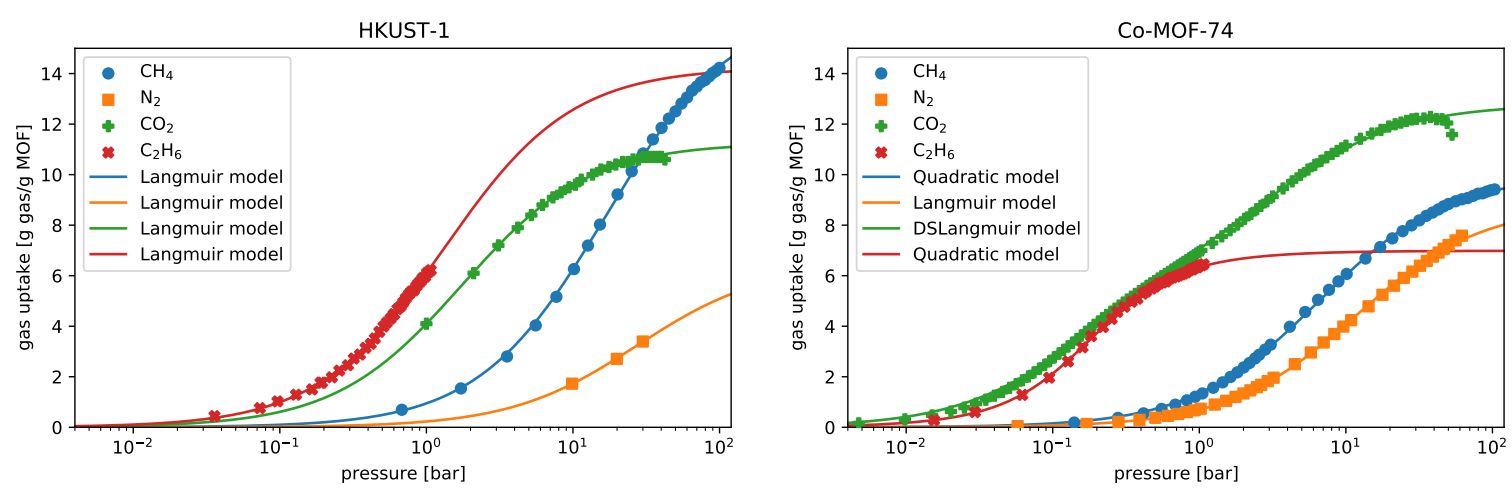

(b)

Figure 4: The two MOFs as candidate recognition elements and their gas adsorption isotherms. (a) The crystal structures of HKUST-1 [32] (left) and Co-MOF-74 [60] (right). (b) Experimentally measured, pure-component $\mathrm{CH}_{4}(298 \mathrm{~K})$ 61, 65], $\mathrm{N}_{2}(298 \mathrm{~K})$ 64, 65], $\mathrm{CO}_{2}(298 \mathrm{~K})$ [62, 65], and $\mathrm{C}_{2} \mathrm{H}_{6}(296 \mathrm{~K})$ [63], and adsorption isotherm data (o, $\square$, +, and $\mathrm{x}$, respectively) in HKUST-1 and Co-MOF-74. Lines show the pure-component adsorption models fit to the data.

\subsection{Case $M=1, G=2$ : single-MOF sensor,} ternary gas mixture

We first consider a single-MOF sensor, either IRMOF-1 or HKUST-1, immersed in a three-component mixture: $\mathrm{CH}_{4} / \mathrm{N}_{2} / \mathrm{CO}_{2}$, $\mathrm{CH}_{4} / \mathrm{N}_{2} / \mathrm{C}_{2} \mathrm{H}_{6}$, or $\mathrm{CH}_{4} / \mathrm{C}_{2} \mathrm{H}_{6} / \mathrm{CO}_{2}$ at 1 bar and $298 \mathrm{~K}$.

Fig. 5 visualizes, under these six scenarios, the adsorption function $m=a(\mathbf{x})$ as well as the unresponsive $\mathbf{v}_{\mathbf{2}}$ (red arrow) and sensitive $\mathbf{v}_{\mathbf{1}}$ (green arrow) directions - given by the right singular vectors of the Jacobian matrix $\mathbf{J}\left(\mathbf{x}_{o p}\right)$ - at a reference gas composition $\mathbf{x}_{o p}$ (black dot). The rows correspond to the three different gas mixtures; the columns correspond to the two different single-MOF sensors. Each panel displays the 2D gas composition space pertaining to the row. The colored lines are contours of the adsorption function $a(\mathbf{x})$ and are labeled by the total adsorbed mass of gas 


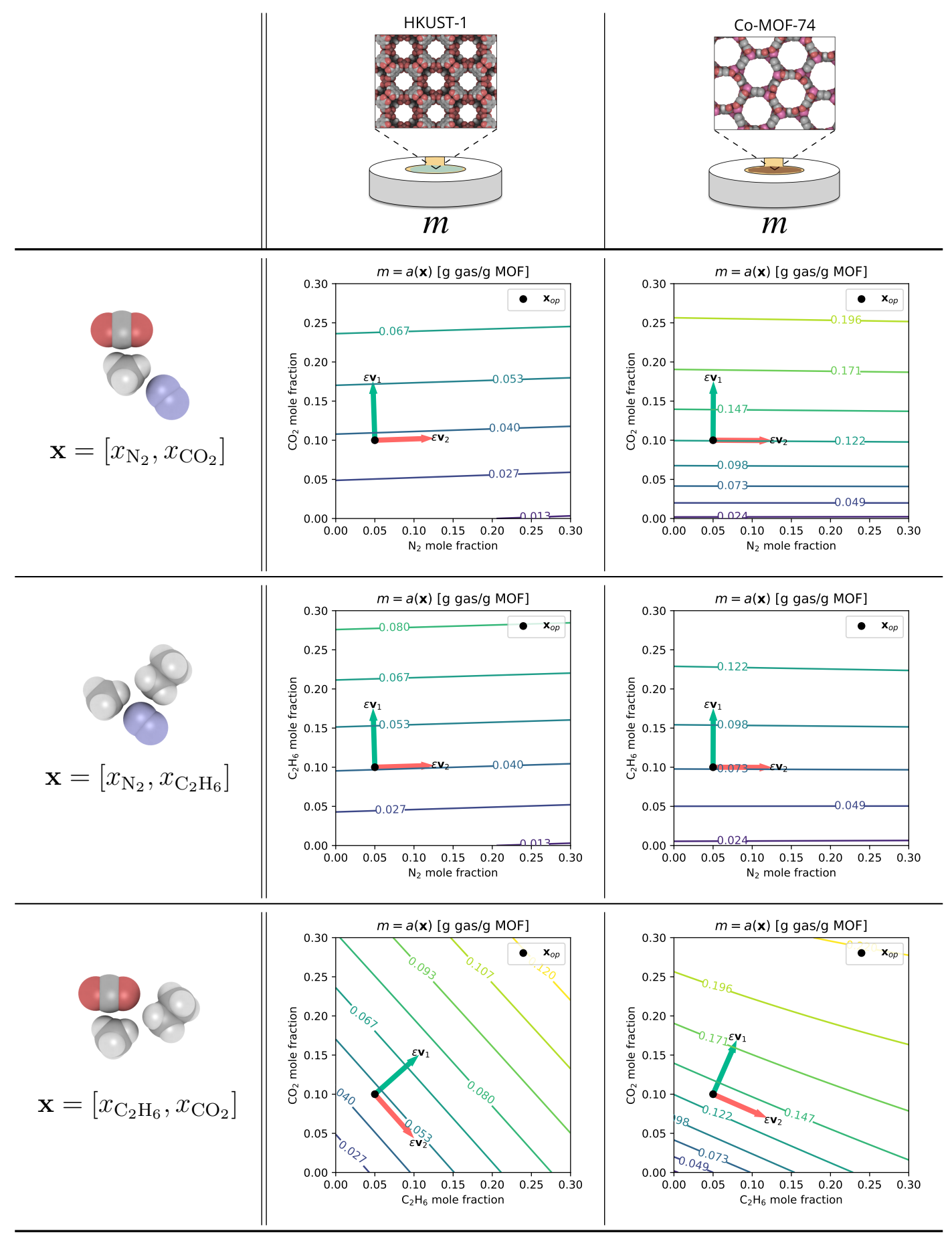

Figure 5: Case $M=1, G=2$ : visualization of $a(\mathbf{x})$ and the unresponsive- and sensitive directions for a single-MOF sensor immersed in a three-component gas mixture at 1 bar and $298 \mathrm{~K}$ under six distinct cases. The row indicates which three species compose the gas mixtures. The column indicates which MOF is used as the recognition element for the gravimetric sensor. In each panel, the plane represents gas composition space. Color-coded contours of the adsorption function $a(\mathbf{x})$ are drawn to indicate unresponsive loci and are labeled with $m=a(\mathbf{x})$ for all $\mathbf{x}$ on that contour. At the operating gas composition $\mathbf{x}_{o p}$, the sensitive (green) and unresponsive (red) directions are shown as $\epsilon \mathbf{v}_{1}$ and $\epsilon \mathbf{V}_{2}$, respectively $(\epsilon=1 / 15)$. 
$m$ in the respective single-MOF sensor. Each map $a: \mathbf{x} \mapsto m$ is clearly non-injective, as an unresponsive locus of points elicits the same sensor response $m$. Within each panel, (i) the non-linearity of the contours and the different areas between successive contours reflect the non-linearity of $a(\mathbf{x})$ and (ii) the slant of the contours reflects the different affinity for the MOF among the three different components of the gas.

The sensitive direction $\mathbf{v}_{1}$ is orthogonal to the contour $\left\{\mathbf{x}: a(\mathbf{x})=m_{o p}\right\}$. Among all fixed-magnitude changes in gas composition, the change in the sensitive direction will elicit the largest response (see eqn. 5). The unresponsive direction is tangential to the contour $\left\{\mathbf{x}: a(\mathbf{x})=m_{o p}\right\}$. The unresponsive subspace is one-dimensional owing to the single constraint imposed by the response of the sensor on the two degrees of freedom in the gas composition. For a small change in gas composition in the unresponsive direction, the sensor response remains approximately the same and, hence, the composition change is not detected by the sensor. Fig. 2 illustrates the underlying physical cause of the unresponsiveness to a composition change, using as an example the HKUST-1 sensor immersed in a $\mathrm{CH}_{4} / \mathrm{C}_{2} \mathrm{H}_{6} / \mathrm{CO}_{2}$ mixture. The gas changes from a dominantly $\mathrm{CH}_{4} / \mathrm{C}_{2} \mathrm{H}_{6}$ to a dominantly $\mathrm{CH}_{4} / \mathrm{CO}_{2}$ mixture, but the mass of adsorbed gas remains constant because the desorption of $\mathrm{C}_{2} \mathrm{H}_{6}$ approximately balances [in mass] the adsorption of $\mathrm{CO}_{2}$ (while $\mathrm{CH}_{4}$ adsorption changes only marginally).

For the two gas mixtures involving $\mathrm{N}_{2}$, the sensitive direction is oriented approximately in the direction of changes in the more readily adsorbing components, $\mathrm{CO}_{2}$ or $\mathrm{C}_{2} \mathrm{H}_{6}$. $\mathrm{N}_{2}$-MOF interactions are weaker than $\mathrm{CO}_{2}$ and $\mathrm{C}_{2} \mathrm{H}_{6}-\mathrm{MOF}$ interactions, reflected in the adsorption isotherms in Fig. 4b. The
$\mathrm{CH}_{4} / \mathrm{C}_{2} \mathrm{H}_{6} / \mathrm{CO}_{2}$ mixture is most interesting, as the sensitive and unresponsive directions each have significant components in the direction of changes in $\mathrm{CO}_{2}$ and $\mathrm{C}_{2} \mathrm{H}_{6}$ mole fractions. Again reflected in the adsorption isotherms in Fig. 4b, $\mathrm{CO}_{2}-\mathrm{MOF}$ and $\mathrm{C}_{2} \mathrm{H}_{6^{-}}$ $\mathrm{MOF}$ interactions are competitive.

Comparing Co-MOF-74 and HKUST-1 for $\mathrm{CH}_{4} / \mathrm{C}_{2} \mathrm{H}_{6} / \mathrm{CO}_{2}$ sensing, the sensitive direction $\mathbf{v}_{1}$ for HKUST-1 has a larger component along $\mathbf{x}=[0.5,0.5]$ (see Fig. 5). Therefore, HKUST-1 is a more suitable sensor for estimating the sum of the $\mathrm{CO}_{2}$ and $\mathrm{C}_{2} \mathrm{H}_{6}$ mole fractions and, thus, the mole fraction of $\mathrm{CH}_{4}$. In other words, the component of the unresponsive direction for HKUST-1 along $\mathbf{x}=$ $[0.5,0.5]$ is smaller than for Co-MOF-74; so, HKUST-1 is not as blind to changes in the $\mathrm{CH}_{4}$ mole fraction in the $\mathrm{CH}_{4} / \mathrm{C}_{2} \mathrm{H}_{6} / \mathrm{CO}_{2}$ mixture than Co-MOF-74. Fig. 6 clarifies: observation of the response $m_{o p}$ of the HKUST-1 sensor places tighter bounds on $x_{\mathrm{CH}_{4}}$ than of the CoMOF-74 sensor because $x_{\mathrm{CH}_{4}}$ is approximately constant along its unresponsive direction. This exemplifies how unresponsive directions and loci could be used to juxtapose two noninjective sensors. However, a more rigorous comparison of the two sensors should include sensitivity to measurement noise [35,50].

\subsection{Case $M=1, G=3$ : single-MOF sensor, quaternary gas mixture}

Now consider a single-MOF sensor - either IRMOF-1 or HKUST-1- immersed in a $\mathrm{CH}_{4} / \mathrm{N}_{2} / \mathrm{CO}_{2} / \mathrm{CH}_{4}$ mixture at 1 bar and $298 \mathrm{~K}$.

Fig. 7 visualizes the adsorption function $m=a(\mathbf{x})$ by showing level sets of it in $3 \mathrm{D}$ gas composition space, colored by the associated response $m$. The black point is the reference gas composition $\mathbf{x}_{o p}$. The sensitive direction 


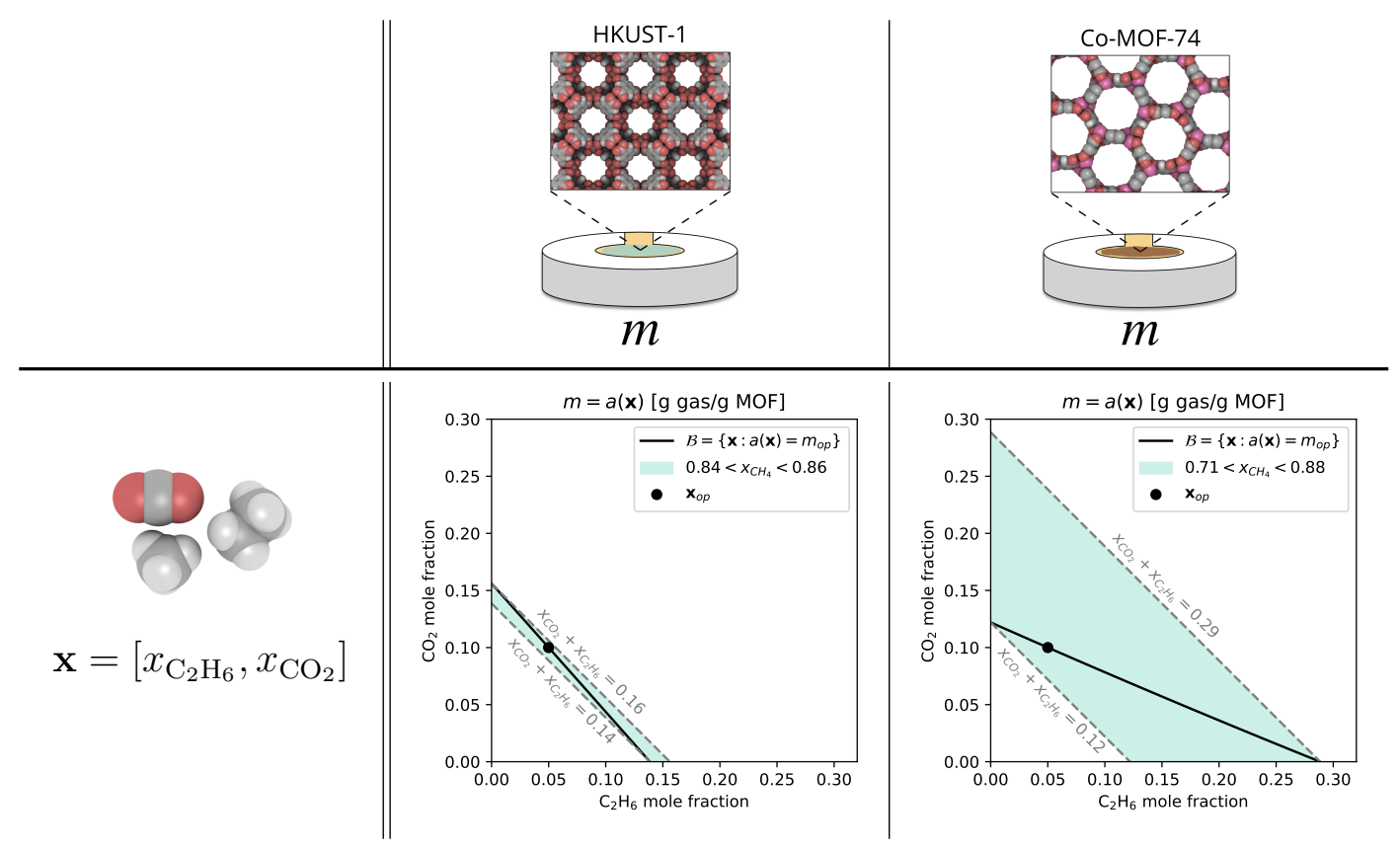

Figure 6: Case $M=1, G=2$ : comparing the utility of two non-injective gas sensors for determining $x_{C_{4}}$ in a $\mathrm{CH}_{4} / \mathrm{C}_{2} \mathrm{H}_{6} / \mathrm{CO}_{2}$ mixture. Along the unresponsive locus $\mathcal{B}$ (black curve) associated with $\mathbf{x}_{o p}$ (black dot), the response of the sensor remains fixed at $m_{o p}$. Observation of the response $m_{o p}$ in each sensor places a lower and upper bound on the mole fraction of $\mathrm{CH}_{4}$, $x_{\mathrm{CH}_{4}}$. The HKUST-1 sensor gives a more useful constraint on $x_{\mathrm{CH}_{4}}$ than the Co-MOF-74 sensor, since $x_{C_{4}}$ is constrained to lie in a smaller interval by the response of the HKUST-1 sensor.

$\mathbf{v}_{1}$ (green vector) is orthogonal to the level set $\left\{\mathbf{x}: a(\mathbf{x})=m_{o p}\right\}$, and the unresponsive subspace, a plane spanned by $\mathbf{v}_{2}$ and $\mathbf{v}_{3}$ (red vectors), is tangent to it. Note the vectors $\mathbf{v}_{2}$ and $\mathbf{v}_{3}$ are not ranked, i.e. one is not more or less unresponsive than the other, and are not unique, in that any two orthonormal vectors that lie in the unresponsive plane can be chosen. The unresponsive subspace is two-dimensional due to the single constraint imposed by the response of the sensor on the three degrees of freedom in the gas composition. Small changes in gas composition on the unresponsive plane are undetectable since the sensor response remains constant. The sensitive direction $\mathbf{v}_{1}$ is orthogonal to the unresponsive plane.

Comparing the sensitive directions of the two sensors at the operating point

$$
\mathbf{x}_{o p}=[0.25,0.2,0.2]
$$

we find:

$$
\begin{aligned}
& \mathbf{v}_{\mathbf{1}} \approx\left[\begin{array}{lll}
-0.02 & 0.67 & 0.74
\end{array}\right] \text { HKUST-1 } \\
& \mathbf{v}_{\mathbf{1}} \approx\left[\begin{array}{lll}
0.02 & 0.96 & 0.27
\end{array}\right] \text { Co-MOF-74. }
\end{aligned}
$$

Both sensors are much more sensitive to changes in $\mathrm{CO}_{2}$ and $\mathrm{C}_{2} \mathrm{H}_{6}$ than to changes in $\mathrm{N}_{2}$. Note the three faces of each cube in Fig. 7] when one mole fraction is set to zero are equivalent to Fig. 5 .

\subsection{Case $M=2, G=3$ : two-MOF sensor array, quaternary gas mixture}

Now consider a two-MOF sensor array, comprised of IRMOF-1 and HKUST-1, immersed in a $\mathrm{CH}_{4} / \mathrm{N}_{2} / \mathrm{CO}_{2} / \mathrm{CH}_{4}$ mixture. See Fig. $8 \mathrm{a}$. 


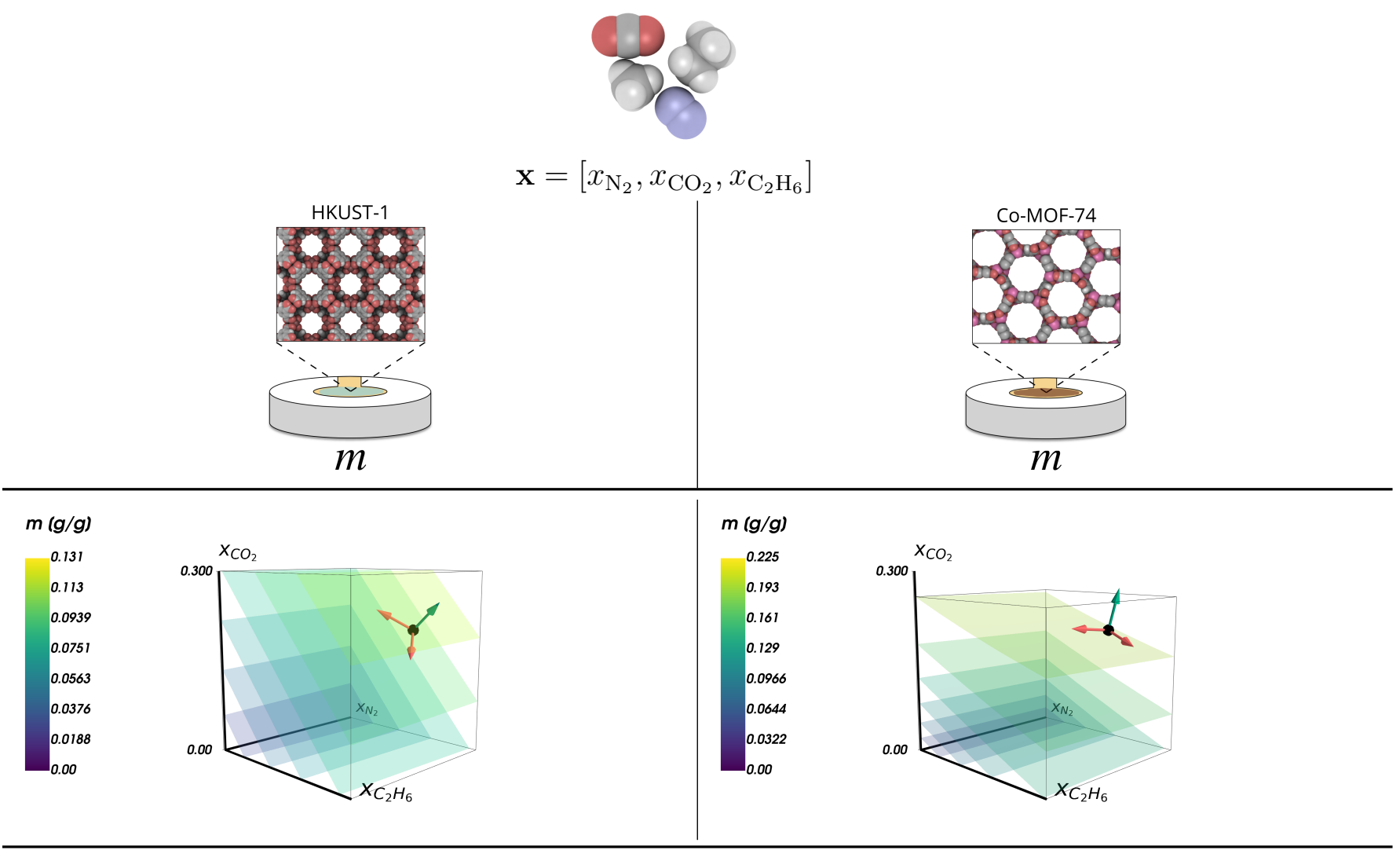

Figure 7: Case $M=1, G=3$ : visualization of $m=a(\mathbf{x})$ and the unresponsive plane and sensitive direction for a single-MOF sensor immersed in a $\mathrm{CH}_{4} / \mathrm{N}_{2} / \mathrm{CO}_{2} / \mathrm{C}_{2} \mathrm{H}_{6}$ gas mixture at 1 bar and $298 \mathrm{~K}$. The column indicates which MOF is used as the recognition element for the gravimetric sensor. Each panel shows gas composition space. Level surfaces of the adsorption function $a(\mathbf{x})$ are color-coded according to the associated $m$. At the operating gas composition $\mathbf{x}_{o p}$ (black point), (i) the sensitive direction $\epsilon \mathbf{v}_{1}$ is shown by the green vector and (ii) the unresponsive plane is spanned by the two unresponsive directions $\epsilon \mathbf{V}_{2}, \epsilon \mathbf{v}_{3}$ shown by the red vectors.

Fig. $8 \mathrm{~b}$ is a visualization to understand the mapping $a: \mathbf{x} \mapsto \mathbf{m}$, with $\mathbf{x}$ in $a$ $3 \mathrm{D}$ gas composition space and $\mathbf{m}$ in a $2 \mathrm{D}$ sensor response space. The image of the sphere of radius $\epsilon=0.04$ in gas composition space (blue, left) is the solid ellipse-like shape (blue, right) in sensor response space; they are (approximately, for the case of the ellipse-like shape) centered, respectively, at the reference gas composition $\mathbf{x}_{o p}$ and its associated response $\mathbf{m}_{o p}$. Unlike the $M=$ $2, G=3$ case in the toy sensor array Fig. $3 \mathrm{~b}$. the image of the sphere is not exactly a solid ellipse owing to the nonlinearity of $a(\mathbf{x})$.

Magnitude- $\epsilon$ changes in the gas composition in the sensitive and intermediate directions in gas composition space - changes of $\epsilon \mathbf{V}_{\mathbf{1}}$ and $\epsilon \mathbf{v}_{2}$, green and yellow vectors in Fig. $8 \mathrm{~b}$ are mapped to the endpoints of the semimajorlike and semiminor-like axes of the ellipse-like shape- $\epsilon \sigma_{1} \mathbf{u}_{\mathbf{1}}$ and $\epsilon \sigma_{2} \mathbf{u}_{2}$, respectively. Among all magnitude- $\epsilon$ changes in the gas composition on the sphere (left), a change $\sim \epsilon \mathbf{v}_{1}$ produces the largest-magnitude sensor array response. 
The sensor array is responsive to, but with reduced sensitivity, changes in the intermediate direction $\epsilon \mathbf{v}_{2}$. On the other hand, the sensor array is unresponsive to small changes in the gas composition in the unresponsive direction of $\mathbf{v}_{3}$. The unresponsive subspace is one dimensional here because the response of the two MOFs places two constraints on the three degrees of freedom in the gas composition.

\section{Non-injective gas sensor arrays are not useless}

The inverse problem [35] of predicting the gas composition $\mathbf{x}$ from the response vector $\mathbf{m}$ of a non-injective gas sensor array typically has infinite solutions, meaning that infinitely many gas compositions are consistent with the response. We therefore cannot make a unique prediction of the gas composition without imposing further assumptions. Despite this, non-injective gas sensor arrays could still be practical.

First, the response of a non-injective sensor arrays places potentially useful constraints on the gas composition. For example, consider the single HKUST-1 sensor in Fig. 5. The response under $\mathrm{CH}_{4} / \mathrm{CO}_{2} / \mathrm{N}_{2}$ mixtures places tight bounds on the mole fraction of $\mathrm{CO}_{2}$ in the mixture. On the other hand, the response under $\mathrm{CH}_{4} / \mathrm{CO}_{2} / \mathrm{C}_{2} \mathrm{H}_{6}$ mixtures cannot delineate between dominantly $\mathrm{CH}_{4} / \mathrm{CO}_{2}$ and $\mathrm{CH}_{4} / \mathrm{C}_{2} \mathrm{H}_{6}$ mixtures (see Fig. 2). Nevertheless, the response does place tight bounds on the sum of the $\mathrm{CO}_{2}$ and $\mathrm{C}_{2} \mathrm{H}_{6}$ mole fractions and, hence, the $\mathrm{CH}_{4}$ mole fraction. See Fig. 6. This tight bound on $x_{\mathrm{CH}_{4}}$ could be very useful and sufficient for certain applications.

Second, imposing additional assumptions about the gas composition can grant the inverse problem a unique solution. Suppose the sensor response changed from $\mathbf{m}_{o p}$, at the operating gas composition $\mathbf{x}_{o p}$, to $\mathbf{m}^{\prime}$. Invoking the linear approximation in eqn. 2, the inverse problem is to find $\mathrm{x}^{\prime}$ that satisfies:

$$
\mathbf{J}\left(\mathbf{x}_{o p}\right)\left(\mathbf{x}^{\prime}-\mathbf{x}_{o p}\right)=\mathbf{m}^{\prime}-\mathbf{m}_{o p}=: \Delta \mathbf{m} .
$$

Because $\mathbf{J}\left(\mathbf{x}_{o p}\right)$ is fat for a non-injective $(M<G)$ gas sensor array, eqn. 16 has infinite solutions (fewer equations than unknowns). However, if we impose an additional assumption that the minimal-L2norm gas composition change $\Delta \mathbf{x}:=\mathbf{x}^{\prime}-\mathbf{x}_{o p}$ from $\mathbf{x}_{o p}$ is the culprit of the change in the sensor response $\Delta \mathbf{m}$, the inverse problem is granted a unique solution. The altered inverse problem:

$$
\min _{\Delta \mathbf{x}: \mathbf{J}\left(\mathbf{x}_{o p}\right) \Delta \mathbf{x}=\Delta \mathbf{m}}\|\Delta \mathbf{x}\|
$$

has a unique solution given by $\Delta \mathbf{x}=\mathbf{J}^{\dagger} \Delta \mathbf{m}$, with $\mathbf{J}^{\dagger}$ the Moore-Penrose psuedo-inverse 59 of J. Again, the SVD in eqn. 6 plays the central role, as the pseudo-inverse can be constructed from it 59 .

\section{Conclusions and Discussion}

Mathematically, gas sensor arrays map each gas composition vector $\mathbf{x}$ to an equilibrium response vector $\mathbf{m}$. For gravimetric, MOFbased gas sensor arrays, this mapping is characterized by a mixed-gas adsorption model $\mathbf{m}=a(\mathbf{x})$. An observation of the [total] mass of gas adsorbed in a MOF generally places one independent constraint $m_{i}=a_{i}(\mathbf{x})$ on the gas composition. We considered when there are fewer MOFs in the array than degrees of freedom in the gas composition (the number of components minus one, given fixed temperature and pressure). As a result, the mapping $a: \mathbf{x} \mapsto \mathbf{m}$ is non-injective (manyto-one). Then, some gas composition changes from $\mathbf{x}_{o p}$ to $\mathbf{x}^{\prime}$ do not cause changes in the 


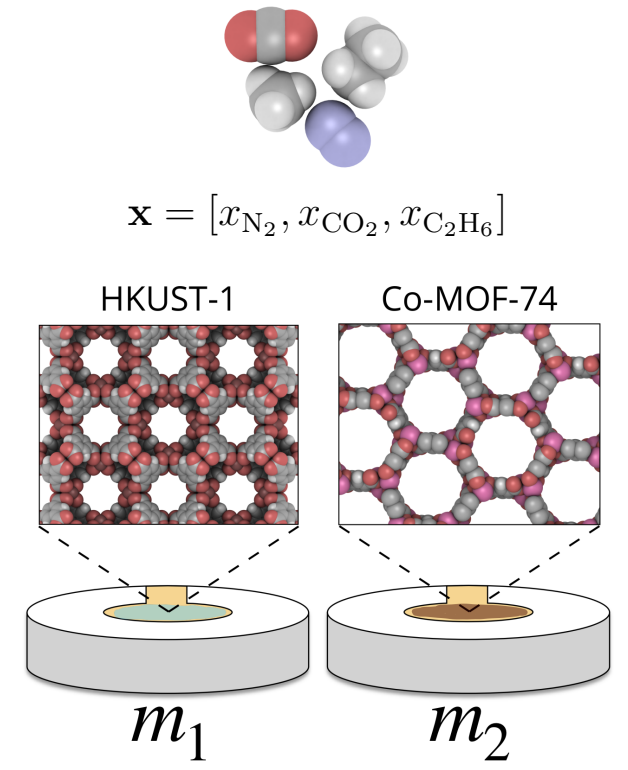

(a)
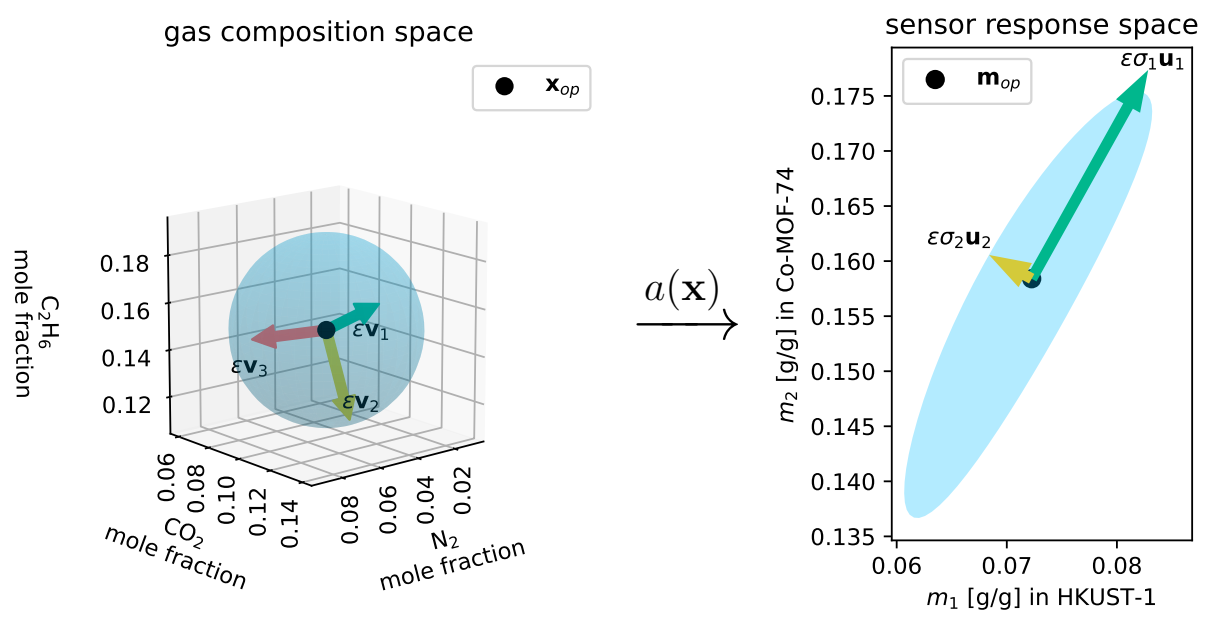

(b)

Figure 8: Case $M=2, G=3$ : visualization of $a(\mathbf{x})$ and unresponsive directions for a two-MOF sensor array immersed in a $\mathrm{CH}_{4} / \mathrm{N}_{2} / \mathrm{CO}_{2} / \mathrm{C}_{2} \mathrm{H}_{6}$ gas mixture at 1 bar and $298 \mathrm{~K}$. (a) The twoMOF sensor array uses HKUST-1 and Co-MOF-74 as recognition elements. (b) The mapping from gas composition space (left) to sensor response space (right) under $a(\mathbf{x})$ for the sensor array in (a). The small sphere of radius $\epsilon=0.04$ in composition space (blue) is mapped to the solid ellipse-like shape (not an exact ellipse like in Fig. $3 \mathrm{~b}$ because $a(\mathbf{x})$ is non-linear) in response space (blue). Under the linear approximant of $a(\mathbf{x}), \tilde{a}(\mathbf{x})$, the vectors $\epsilon \mathbf{v}_{2}, \epsilon \mathbf{V}_{2}, \epsilon \mathbf{v}_{3}$ are mapped to $\epsilon \sigma_{1} \mathbf{u}_{1}, \epsilon \sigma_{2} \mathbf{u}_{2}, \mathbf{0}$, respectively. The unresponsive direction is $\mathbf{v}_{\mathbf{3}}$ (red) and the sensitive direction is $\mathbf{v}_{1}$ (green).

sensor response $\mathbf{m}_{o p}$ despite $\mathbf{x}^{\prime} \neq \mathbf{x}_{o p}$ and determine the local unresponsive subspace of thus are undetectable. We showed how to gas composition space from the null space 
of the Jacobian matrix $\mathbf{J}$ of the adsorption model $a(\mathbf{x})$. Whereupon small changes in gas composition in an unresponsive direction, the sensor response remains constant. More, the right singular vectors of $\mathbf{J}$ associated with its non-zero singular values give directions in gas compositions to which the sensor is responsive, ranked by sensitivity. We demonstrated the identification of unresponsive and ranked responsive directions in non-injective, singleand double-MOF sensor arrays based on HKUST-1 and Co-MOF-74 immersed in a mixture involving $\mathrm{CH}_{4}, \mathrm{CO}_{2}, \mathrm{C}_{2} \mathrm{H}_{6}$, and $\mathrm{N}_{2}$ relevant to natural gas sensing [45]. Our case studies involved $\leq$ two MOFs and $\leq$ four components in the gas, instructively allowing us to visualize $a(\mathbf{x})$ and clarify the meaning of the unresponsive and ranked responsive directions in gas composition space. The SVD in eqn. 6 is particularly powerful for large sensor arrays and high-dimensional gas composition spaces where, unfortunately, visualization of $a(\mathbf{x})$ is extremely difficult.

Unsurprisingly, the identification of unresponsive and ranked responsive directions have analogies in multiple-input, multiple-output control systems: observability $[67-69]$ and directionality 70,71 , respectively.

Why is it useful to identify unresponsive directions in gas sensors? A gas sensor is unresponsive to and thus cannot detect gas composition changes in the unresponsive direction. Determining the unresponsive directions of a gas sensor is important to quantify its limitations and identify its vulnerabilities to adversarial attacks [44]. In addition, given a set of candidate non-injective gas sensors for a sensing task, we may be able rank them according to their unresponsive directions e.g. having a minimal component along a pertinent direction.
Does the notion and identification of unresponsive directions generalize beyond gravimetric, MOF-based sensors? The notion and process of identifying unresponsive directions herein generalizes beyond the specific sensing mechanism of observing the total mass of adsorbed gas in a MOF. To adapt to a generic array of sensing elements, each exhibiting some observable property dependent on the gas composition, redefine:

- $\mathbf{m}$ as the vector containing the observed properties of the $M$ sensing elements in response to the gas composition $\mathbf{x}$.

- $a(\mathbf{x})$ as a function that models the response $\mathbf{m}$ to each gas composition $\mathbf{x}$.

Even without much understanding of the underlying physics linking the response to the gas composition, we could build the model $a(\mathbf{x})$ as follows. Construct the sensor array. Conduct gas exposure experiments: observe the response over a grid of gas compositions to generate data $\mathcal{D}=\left\{\left(\mathbf{x}_{i}, \mathbf{m}_{i}\right)\right\}$. Build a regression model of $a(\mathbf{x})$ using the training data $\mathcal{D}$.

Gravimetric MOF sensors are attractive from a modeling perspective because we can construct a reasonable model $a(\mathbf{x})$ without actually constructing the sensor array and conducting laborious experiments. Gas adsorption data in MOFs is abundant; thermodynamic theories of gas adsorption are welldeveloped; and molecular models and simulations can predict adsorption in MOFs. The latter enables the computational design of MOFbased gas sensor arrays, as exemplified by Wilmer and coworkers 49,50, who use molecular simulations of adsorption to predict the response of gravimetric, MOF-based sensor arrays and then evaluate their fitness for gas sensing. 
How can we remedy non-injective gas sensors to give a determined inverse problem? The observed response of a non-injective gas sensor generally places an insufficient number of constraints on the gas composition to give the inverse problem a unique solution. We can make a non-injective sensor array injective by (i) retrofitting it with additional sensing elements (MOFs), (ii) designing sensing elements (MOFs) that do not respond appreciably to interferents in the gas that we are not concerned about, or (iii) imposing additional assumptions on the gas composition as in eqn. 17 .

Is an injective sensor array the end goal? The inverse problem associated with an injective sensor array will theoretically always have a unique solution. However, in practice, the measurements of the masses of gas adsorbed in each MOF are corrupted by measurement error. As a result, the applied inverse problem 72] for an injective sensor array could still (i) have no solution or (ii) be ill-conditioned, where small measurement errors lead to large changes in the predicted gas composition [35]. The conditioning of the inverse problem, colloquially speaking, depends on the diversity of the MOFs in the array and their interactions with the components of the gas. One method to evaluate the fitness of different injective sensor arrays is to analyze the conditioning of their inverse problem [35.

Future work remains. First, we posed the gas sensing problem in terms of the equilibrium response of the gas sensor. However, the mass of gas adsorbed in a MOF is generally dynamic; gas must enter into and diffuse in the thin film of the MOF attached to the QCM 73 75]. Future work entails (i) considering the diffusion kinetics when evaluating MOF-based sensors to avoid slow response times, (ii) posing inverse problems in terms of the dynamic response of the sensor, as in Rajagopalan and Petit [52], and (ii) extracting additional information about the gas composition from the dynamics of the response.

Second, we assumed isothermal conditions. We may wish to use a gas sensor to robustly predict the gas composition in a range of temperatures. Future work entails building an adsorption model $a(\mathbf{x} ; T)$ that applies under different temperatures $T$, then (a) inputting a separate measurement of $T$ or (b) determining $T$ as part of the inverse problem.

Third, imposing additional assumptions on the gas composition can endow the underdetermined inverse problem associated with a non-injective gas sensor with a unique solution. This idea of imposing additional assumptions, consistent with but not imposed by the physics underlying the problem, is a common approach to, given an inverse problems with nonunique solutions, grant it a unique solution [72]. We provided one example in eqn. 17, where we sought the minimumnorm change in the gas composition consistent with the change in the response.

\section{Acknowledgements.}

We acknowledge support from the U.S. Department of Defense (DoD) Defense Threat Reduction Agency (HDTRA-19-31270) (NG, CMS, PT) and the National Science Foundation (NSF) (award 1920945) (AH, CMS, XF).

\section{References}

[1] Wenwen Hu, Liangtian Wan, Yingying Jian, Cong Ren, Ke Jin, Xinghua Su, Xiaoxia Bai, Hossam Haick, Mingshui Yao, and Weiwei Wu. Electronic noses: From advanced materials to sensors aided with data processing. Advanced $M a-$ 
terials Technologies, page 1800488, December 2018.

[2] Prashant Kumar, Lidia Morawska, Claudio Martani, George Biskos, Marina Neophytou, Silvana Di Sabatino, Margaret Bell, Leslie Norford, and Rex Britter. The rise of lowcost sensing for managing air pollution in cities. Environment International, 75:199-205, February 2015.

[3] Gady Konvalina and Hossam Haick. Sensors for breath testing: from nanomaterials to comprehensive disease detection. Accounts of Chemical Research, 47(1):66-76, 2014.

[4] Hanie Yousefi, Hsuan-Ming Su, Sara M Imani, Kais Alkhaldi, Carlos D M. Filipe, and Tohid F Didar. Intelligent food packaging: A review of smart sensing technologies for monitoring food quality. ACS Sensors, 4(4):808-821, 2019.

[5] Timur Islamoglu, Zhijie Chen, Megan C. Wasson, Cassandra T. Buru, Kent O. Kirlikovali, Unjila Afrin, Mohammad Rasel Mian, and Omar K. Farha. Metal-organic frameworks against toxic chemicals. Chemical Reviews, 120(16):81308160, March 2020.

[6] Rama Sesha Vemuri and Paul Armatis. An overview of detection and neutralization of chemical warfare agents using metal organic frameworks. Journal of Bioterrorism \&3 Biodefense, 06(03), 2015.

[7] Alphus Wilson. Diverse applications of electronicnose technologies in agriculture and forestry. Sensors, 13(2):2295-2348, February 2013.

[8] Hiroyasu Furukawa, Kyle E. Cordova, Michael O'Keeffe, and Omar M. Yaghi. The chemistry and applications of metal-organic frameworks. Science, 341(6149):1230444, August 2013.

[9] Lauren E Kreno, Kirsty Leong, Omar K Farha, Mark Allendorf, Richard P Van Duyne, and Joseph T Hupp. Metal-organic framework materials as chemical sensors. Chemical Reviews, 112(2):1105-1125, 2011.

[10] Dominic J Wales, Julien Grand, Valeska P Ting, Richard D Burke, Karen J Edler, Chris R Bowen, Svetlana Mintova, and Andrew D Burrows. Gas sensing using porous materials for automotive applications. Chemical Society Reviews, 44(13):4290-4321, 2015.

[11] Hai-Yang Li, Shu-Na Zhao, Shuang-Quan Zang, and Jing Li. Functional metal-organic frameworks as effective sensors of gases and volatile compounds. Chemical Society Reviews,
49(17):6364-6401, 2020.

[12] Leo J. Small, Mara E. Schindelholz, and Tina M. Nenoff. Hold on tight: MOFbased irreversible gas sensors. Industrial \&6 Engineering Chemistry Research, May 2021.

[13] MR Tchalala, PM Bhatt, KN Chappanda, SR Tavares, K Adil, Y Belmabkhout, A Shkurenko, A Cadiau, N Heymans, G De Weireld, G Maurin, KN Salama, and M Eddaoudi. Fluorinated mof platform for selective removal and sensing of $\mathrm{so}_{2}$ from flue gas and air. Nature Communications, 10(1):1328, 2019.

[14] Michael G Campbell, Sophie F Liu, Timothy M Swager, and Mircea Dinca. Chemiresistive sensor arrays from conductive $2 \mathrm{~d}$ metal-organic frameworks. Journal of the American Chemical Society, 137(43):13780-13783, 2015.

[15] Zheng Meng, Aylin Aykanat, and Katherine A. Mirica. Welding metallophthalocyanines into bimetallic molecular meshes for ultrasensitive, low-power chemiresistive detection of gases. Journal of the American Chemical Society, 141(5):2046-2053, December 2018.

[16] Andrew M. Ullman, Christopher G. Jones, F. Patrick Doty, Vitalie Stavila, A. Alec Talin, and Mark D. Allendorf. Hybrid polymer/metal-organic framework films for colorimetric water sensing over a wide concentration range. ACS Applied Materials \& Interfaces, 10(28):24201-24208, June 2018.

[17] Rui-Biao Lin, Si-Yang Liu, Jia-Wen Ye, Xu-Yu Li, and Jie-Peng Zhang. Photoluminescent metalorganic frameworks for gas sensing. Advanced Science, 3(7):1500434, 2016.

[18] William P. Lustig, Soumya Mukherjee, Nathan D. Rudd, Aamod V. Desai, Jing Li, and Sujit K. Ghosh. Metal-organic frameworks: functional luminescent and photonic materials for sensing applications. Chemical Society Reviews, 46(11):3242-3285, 2017.

[19] Mark D Allendorf, Ronald JT Houk, Leanne Andruszkiewicz, A Alec Talin, Joel Pikarsky, Arnab Choudhury, Kenneth A Gall, and Peter J Hesketh. Stress-induced chemical detection using flexible metal- organic frameworks. Journal of the American Chemical Society, 130(44):14404-14405, 2008.

[20] Hamish Yeung, G Yoshikawac, and K Shibab. Strain-based chemical sensing using metalorganic framework nanoparticles. Journal of 
Materials Chemistry A, 8:18007-18014, 2020.

[21] Osama Shekhah, J Liu, RA Fischer, and Ch Wöll. Mof thin films: existing and future applications. Chemical Society Reviews, 40(2):1081-1106, 2011.

[22] Ivo Stassen, Nicholas Burtch, Alec Talin, Paolo Falcaro, Mark Allendorf, and Rob Ameloot. An updated roadmap for the integration of metalorganic frameworks with electronic devices and chemical sensors. Chemical Society Reviews, 46(11):3185-3241, 2017.

[23] Anna Lisa Semrau, Zhenyu Zhou, Soumya Mukherjee, Min Tu, Weijin Li, and Roland A. Fischer. Surface-mounted metal-organic frameworks: Past, present, and future perspectives. Langmuir, June 2021.

[24] Hiroki Yamagiwa, Seiko Sato, Tadashi Fukawa, Tsuyoshi Ikehara, Ryutaro Maeda, Takashi Mihara, and Mutsumi Kimura. Detection of volatile organic compounds by weightdetectable sensors coated with metal-organic frameworks. Scientific Reports, 4:6247, 2014.

[25] Min Tu, Suttipong Wannapaiboon, Kira Khaletskaya, and Roland A Fischer. Engineering zeolitic-imidazolate framework (zif) thin film devices for selective detection of volatile organic compounds. Advanced Functional Materials, 25(28):4470-4479, 2015.

[26] Mohamed Rachid Tchalala, Youssef Belmabkhout, Karim Adil, Karumbaiah Nanaiah Chappanda, Amandine Cadiau, Prashant M Bhatt, Khaled Nabil Salama, and Mohamed Eddaoudi. Concurrent Sensing of $\mathrm{CO}_{2}$ and $\mathrm{H}_{2} \mathrm{O}$ from Air Using Ultramicroporous Fluorinated Metal-Organic Frameworks: Effect of Transduction Mechanism on the Sensing Performance. ACS Applied Materials \& Interfaces, 11(1):1706-1712, 2018.

[27] Salih Okur, Peng Qin, Abhinav Chandresh, Chun Li, Zejun Zhang, Ulrich Lemmer, and Lars Heinke. An enantioselective e-nose: An array of nanoporous homochiral MOF films for stereospecific sensing of chiral odors. Angewandte Chemie International Edition, 60(7):3566-3571, December 2020.

[28] S. Okur, C. Li, Z. Zhang, S. Vaidurya Pratap, M. Sarheed, A. Kanbar, L. Franke, F. Geislhöringer, L. Heinke, U. Lemmer, P. Nick, and C. Wöll. Sniff species - surmof based sensor array discriminate aromatic plants beyond the genus level. Preprints, 2021.
[29] Vitalie Stavila, Christian Schneider, Curtis Mowry, Todd R Zeitler, Jeffery A Greathouse, Alex L Robinson, Julie M Denning, Joanne Volponi, Kirsty Leong, William Quan, Min Tu, Roland A. Fischer, and Mark D. Allendorf. Thin film growth of nbo MOFs and their integration with electroacoustic devices. Advanced Functional Materials, 26(11):1699-1707, 2016.

[30] Benjamin Paschke, Achim Wixforth, Dmytro Denysenko, and Dirk Volkmer. Fast surface acoustic wave-based sensors to investigate the kinetics of gas uptake in ultra-microporous frameworks. ACS Sensors, 2(6):740-747, 2017.

[31] Jagannath Devkota, Ki-Joong Kim, Paul R Ohodnicki, Jeffrey T Culp, David W Greve, and Jonathan W Lekse. Zeolitic imidazolate framework-coated acoustic sensors for room temperature detection of carbon dioxide and methane. Nanoscale, 10(17):8075-8087, 2018.

[32] Stephen S-Y Chui, Samuel M-F Lo, Jonathan PH Charmant, A Guy Orpen, and Ian D Williams. A chemically functionalizable nanoporous material $\left[\mathrm{Cu}_{3}(\mathrm{TMA})_{2}\left(\mathrm{H}_{2} \mathrm{O}\right)_{3}\right]_{n}$. Science, 283(5405):1148-1150, 1999.

[33] Luyu Wang. Metal-organic frameworks for QCMbased gas sensors: A review. Sensors and Actuators A: Physical, 307:111984, June 2020.

[34] Xiao Liu, Sitian Cheng, Hong Liu, Sha Hu, Daqiang Zhang, and Huansheng Ning. A survey on gas sensing technology. Sensors, 12(7):96359665, 2012.

[35] Rachel Sousa and Cory M. Simon. Evaluating the fitness of combinations of adsorbents for quantitative gas sensor arrays. ACS Sensors, 5(12):4035-4047, December 2020.

[36] Arni Sturluson, Rachel Sousa, Yujing Zhang, Melanie T. Huynh, Caleb Laird, Arthur H. P. York, Carson Silsby, Chih-Hung Chang, and Cory M. Simon. Curating metal-organic frameworks to compose robust gas sensor arrays in dilute conditions. ACS Applied Materials \& Interfaces, 12(5):6546-6564, January 2020.

[37] Wenwen $\mathrm{Hu}$, Liangtian Wan, Yingying Jian, Cong Ren, Ke Jin, Xinghua Su, Xiaoxia Bai, Hossam Haick, Mingshui Yao, and Weiwei Wu. Electronic noses: from advanced materials to sensors aided with data processing. Advanced Materials Technologies, 4(2):1800488, 2019.

[38] Keith J Albert, Nathan S Lewis, Caroline L Schauer, Gregory A Sotzing, Shannon E Stitzel, Thomas P Vaid, and David R Walt. Cross- 
reactive chemical sensor arrays. Chemical Reviews, 100(7):2595-2626, 2000.

[39] Julian W. Gardner and Philip N. Bartlett. A brief history of electronic noses. Sensors and Actuators B: Chemical, 18(1-3):210-211, March 1994.

[40] P. C. Jurs, G. A. Bakken, and H. E. McClelland. Computational methods for the analysis of chemical sensor array data from volatile analytes. Chemical Reviews, 100(7):2649-2678, June 2000.

[41] Salih Okur, Zejun Zhang, Mohammed Sarheed, Peter Nick, Uli Lemmer, and Lars Heinke. Towards a MOF e-nose: A SURMOF sensor array for detection and discrimination of plant oil scents and their mixtures. Sensors and Actuators B: Chemical, 306:127502, March 2020.

[42] Tu Lee, Zheng Xin Liu, and Hung Lin Lee. A biomimetic nose by microcrystals and oriented films of luminescent porous metal-organic frameworks. Crystal Growth 8 Design, 11(9):4146-4154, September 2011.

[43] Michael Ko, Aylin Aykanat, Merry K Smith, and Katherine A Mirica. Drawing sensors with ballmilled blends of metal-organic frameworks and graphite. Sensors, 17(10):2192, 2017.

[44] Battista Biggio and Fabio Roli. Wild patterns: Ten years after the rise of adversarial machine learning. Pattern Recognition, 84:317-331, 2018.

[45] S. Faramawy, T. Zaki, and A.A.-E. Sakr. Natural gas origin, composition, and processing: A review. Journal of Natural Gas Science and Engineering, 34:34-54, August 2016.

[46] Alan L Myers and John M Prausnitz. Thermodynamics of mixed-gas adsorption. AIChE journal, 11(1):121-127, 1965.

[47] Krista S Walton and David S Sholl. Predicting multicomponent adsorption: 50 years of the ideal adsorbed solution theory. AIChE Journal, 61(9):2757-2762, 2015.

[48] Jenna Ann Gustafson and Christopher E Wilmer. Intelligent selection of metal-organic framework arrays for methane sensing via genetic algorithms. ACS Sensors, 4(6):1586-1593, 2019.

[49] Jenna A Gustafson and Christopher E Wilmer. Computational design of metal-organic framework arrays for gas sensing: Influence of array size and composition on sensor performance. The Journal of Physical Chemistry C,
121(11):6033-6038, 2017.

[50] Jenna A Gustafson and Christopher E Wilmer. Optimizing Information Content in MOF Sensor Arrays for Analyzing Methane-air Mixtures. Sensors and Actuators B: Chemical, 267:483493, 2018.

[51] Brian A Day and Christopher E Wilmer. Genetic Algorithm Design of MOF-based Gas Sensor Arrays for $\mathrm{CO}_{2}$-in-Air Sensing. Sensors, 20(3):924, 2020.

[52] Ashwin Kumar Rajagopalan and Camille Petit. Material screening for gas sensing using an electronic nose: Gas sorption thermodynamic and kinetic considerations. ChemRxiv, May 2021.

[53] T.C. Pearce. Odor to sensor space transformations in biological and artificial noses. Neurocomputing, 32-33:941-952, June 2000.

[54] Hans Swenson and Nicholas P. Stadie. Langmuir's theory of adsorption: A centennial review. Langmuir, 35(16):5409-5426, March 2019.

[55] A. L. Myers and J. M. Prausnitz. Thermodynamics of mixed-gas adsorption. AIChE Journal, 11(1):121-127, January 1965.

[56] Arni Sturluson, Melanie T. Huynh, Alec R. Kaija, Caleb Laird, Sunghyun Yoon, Feier Hou, Zhenxing Feng, Christopher E. Wilmer, Yamil J. Colón, Yongchul G. Chung, Daniel W. Siderius, and Cory M. Simon. The role of molecular modelling and simulation in the discovery and deployment of metal-organic frameworks for gas storage and separation. Molecular Simulation, 45(14-15):1082-1121, August 2019.

[57] David Dubbeldam, Ariana Torres-Knoop, and Krista S. Walton. On the inner workings of monte carlo codes. Molecular Simulation, 39(14-15):1253-1292, December 2013.

[58] Dan Kalman. A singularly valuable decomposition: the svd of a matrix. The college mathematics journal, 27(1):2-23, 1996.

[59] Gilbert Strang. Introduction to Linear Algebra, volume 5. Wellesley-Cambridge Press Wellesley, MA, 2016.

[60] Pascal D. C. Dietzel, Yusuke Morita, Richard Blom, and Helmer Fjellvåg. An in situ high-temperature single-crystal investigation of a dehydrated metal-organic framework compound and field-induced magnetization of onedimensional metal-oxygen chains. Angewandte Chemie International Edition, 44(39):63546358, October 2005. 
[61] Jarad A. Mason, Mike Veenstra, and Jeffrey R. Long. Evaluating metal-organic frameworks for natural gas storage. Chem. Sci., 5(1):32-51, 2014.

[62] Andrew R. Millward and Omar M. Yaghi. Metalorganic frameworks with exceptionally high capacity for storage of carbon dioxide at room temperature. Journal of the American Chemical Society, 127(51):17998-17999, December 2005.

[63] Yabing He, Rajamani Krishna, and Banglin Chen. Metal-organic frameworks with potential for energy-efficient adsorptive separation of light hydrocarbons. Energy \& Environmental Science, 5(10):9107, 2012.

[64] J. Rother and T. Fieback. Multicomponent adsorption measurements on activated carbon, zeolite molecular sieve and metal-organic framework. Adsorption, 19(5):1065-1074, March 2013.

[65] Decai Yu, A. Ozgur Yazaydin, Joseph R. Lane, Pascal D. C. Dietzel, and Randall Q. Snurr. A combined experimental and quantum chemical study of $\mathrm{CO} 2$ adsorption in the metal-organic framework CPO-27 with different metals. Chemical Science, 4(9):3544, 2013.

[66] Cory M Simon, Berend Smit, and Maciej Haranczyk. pyIAST: Ideal adsorbed solution theory (IAST) Python package. Computer Physics Communications, 200:364-380, 2016.

[67] E. Castillo, A.J. Conejo, R.E. Pruneda, and C. Solares. State estimation observability based on the null space of the measurement jacobian matrix. IEEE Transactions on Power Systems, 20(3):1656-1658, August 2005.

[68] Yang-Yu Liu, Jean-Jacques Slotine, and AlbertLászló Barabási. Observability of complex systems. Proceedings of the National Academy of Sciences, 110(7):2460-2465, 2013.

[69] Elmer G Gilbert. Controllability and observability in multivariable control systems. Journal of the Society for Industrial and Applied Mathematics, Series A: Control, 1(2):128-151, 1963.

[70] Sigurd Skogestad and Ian Postlethwaite. Multivariable feedback control: analysis and design, volume 2. Citeseer, 2007.

[71] Henrik Weisberg Andersen and Mogens Kümmel. Evaluating estimation of gain directionality. Journal of Process Control, 2(2):59-66, January 1992.

[72] Pierre C. Sabatier. Past and future of inverse problems. Journal of Mathematical Physics, 41(6):4082-4124, June 2000.

[73] Lars Heinke and Christof Wöll. Adsorption and diffusion in thin films of nanoporous metalorganic frameworks: ferrocene in SURMOF $\mathrm{Cu}_{2}$ (ndc) $)_{2}$ (dabco). Physical Chemistry Chemical Physics, 15(23):9295-9299, 2013.

[74] Lars Heinke, Zhigang Gu, and Christof Wöll. The surface barrier phenomenon at the loading of metal-organic frameworks. Nature Communications, 5(1):1-6, 2014.

[75] Tobias Titze, Alexander Lauerer, Lars Heinke, Christian Chmelik, Nils ER Zimmermann, Frerich J Keil, Douglas M Ruthven, and Jörg Kärger. Transport in nanoporous materials including MOFs: The applicability of Fick's laws. Angewandte Chemie International Edition, 54(48):14580-14583, 2015. 